\title{
SEEING FASHION THROUGH SOUND
}

\author{
by \\ Jenni Lin Armstrong \\ Bachelor of Arts, Montreal, Quebec, 2005 \\ Diploma of Collegial Studies, Montreal, 2002
}

\author{
A Major Research Project \\ presented to Ryerson University \\ in partial fulfillment of the \\ requirements for the degree of \\ Master of Arts \\ in the Program of \\ Fashion
}

Toronto, Ontario, Canada, 2015

(C) Jenni Lin Armstrong 2015 


\section{AUTHOR'S DECLARATION FOR ELECTRONIC SUBMISSION OF A MRP}

I hereby declare that I am the sole author of this MRP. This is a true copy of the MRP, including any required final revisions.

I authorize Ryerson University to lend this MRP to other institutions or individuals for the purpose of scholarly research.

I further authorize Ryerson University to reproduce this MRP by photocopying or by other means, in total or in part, at the request of other institutions or individuals for the purpose of scholarly research.

I understand that my MRP may be made electronically available to the public. 


\section{Abstract \\ SEEING FASHION THROUGH SOUND \\ Jenni Lin Armstrong \\ Master of Arts in Fashion, 2015 \\ Ryerson University}

The 2005 Accessibility for Ontarians with Disabilities Act (AODA) has legislated museums to amend tangible and intangible barriers within their curatorial practices by 2025 . In this study, a Métis researcher-practitioner explored artistic ways that museums might curate direct and accessible experiences with artefacts through wearable technology. Utilizing a practice-led creative process, non-traditional aboriginal regalia was developed and displayed in a multimedia installation. The artifact was inspired by the Ojibwe Jingle Dress and dance, which empower and heal through sound. To augment the exhibition experience, a wearable audio system enhances sound from the Jingle Dress and touchless elements, such as electromagnetically induced sound, created an environment where visitor interaction would not compromise artefact preservation. A sound experience was only accessible if a visitor learned how to respectfully interact with the artefact. Both artefact and installation serve as recommendations for museums to effectuate inclusive exhibition experiences and address AODA requirements. 


\section{Acknowledgements}

The artist wishes to express her deepest gratitude and appreciation for the kind supervision and advice of Professors Grahame Lynch and Sandra Tullio-Pow of the Graduate School of Fashion at Ryerson University, Toronto, Canada. Throughout each stage of this research process the artist received guidance, helpful suggestions, and continuous support from these professors, all which have contributed greatly to her academic success.

Many thanks presented to the Ryerson Aboriginal Student Services at Ryerson University, for not only providing useful discussion, knowledge, and moral and spiritual support during the research process, but also for providing academic assistance and the funding that was necessary to build the installation components.

Special thanks to Sherri Fetterly and Gavin O’Hara for their help proofreading and editing the text. Thank you also to Jonathan Drolet. These friends have provided continuous encouragement, motivation, and support.

Finally, many thanks to technical help offered voluntarily by members of Hacklab, Toronto and the Hobbyist Electronics Club of Ryerson University's Engineering Department. 


\section{Table of Contents}

Page

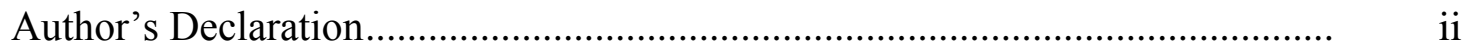

Abstract ............................................................................................. ii

Acknowledgments................................................................................ iv

List of Tables .................................................................................... viii

List of Figures ........................................................................................ ix

CHAPTER 1: INTRODUCTION ......................................................................

1.1 Museum Policy for Restriction and Access to Physical Interactions......... 1

1.2 Research Aims of this Study .................................................................. 2

1.3 Planning for the Accessibility Mandate for Ontarians with Disabilities.... 3

1.4 A First Nations Approach to Cultural Preservation Through Interaction .... 4

CHAPTER 2: LITERATURE REVIEW …………………………………...... 7

2.1 Curatorial Challenges ..............................................................................

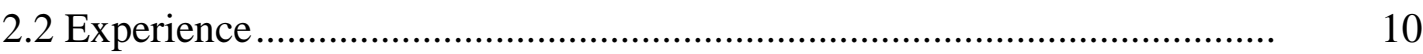

2.3 Technology-Enabled Exhibitions ............................................................... 14

2.4 Interactive Sound Installations ......................................................................... 18

CHAPTER 3: METHODOLOGY AND METHODS .......................................... 21

3.1 Practice-Led, Indigenous Artistic Research Process.................................. 21

3.2 Regalia as a Symbol of Curatorial Struggle ............................................... 24

3.3 Interaction ................................................................................... 26

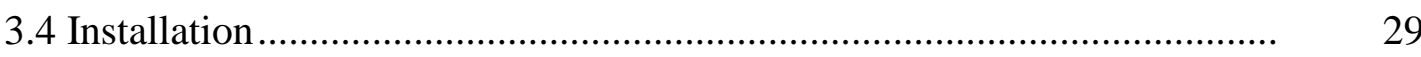

CHAPTER 4: RESEARCH PROCESS - A CHRONOLOGY ........................... 32

4.1 Wearable Audio Technology Research...................................................... 33

4.2 Research and Execution of Jingle Dress Regalia ........................................ $\quad 39$

4.3 Working with Consultants: Evolution of the Wearable Audio

Electronic System ……….................................................................. 44

4.4 Electronic Audio Amplification ................................................................ 45

4.5 Digital Recordings ............................................................................ 4

4.6 Handheld Magnetic Cone.......................................................................... 49

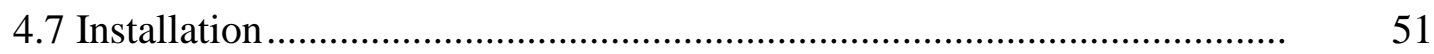

4.7.1 Staging the Environment ............................................................... 52

4.7.2 Connecting with the Audience ...................................................... 55

4.7.3 Observable Interactions ............................................................... 56

CHAPTER 5: RECOMMENDATIONS AND CONCLUSION .......................... 59

5.1 Limitations of the Study ........................................................................ 5

5.2 Summary of Study Results........................................................................ 61

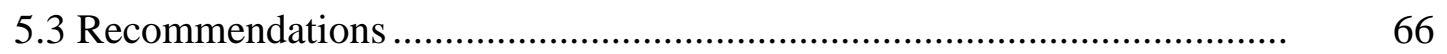

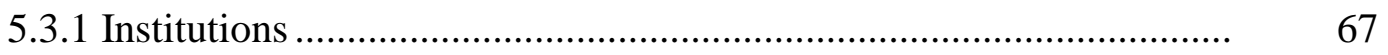




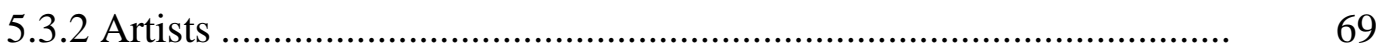

5.3.3 Visitors ................................................................................. $\quad 70$

5.4 Implementation Challenges and Areas of Future Research ....................... 71

Appendix: Figures.......................................................................................... 73

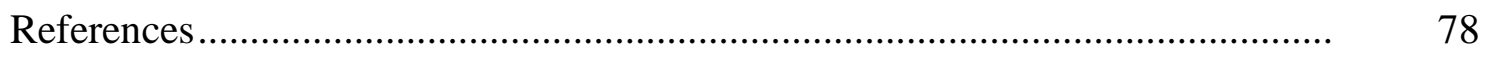




\section{List of Tables}

$\begin{array}{lll}\text { Table } & \text { Page }\end{array}$

1: Conductive Filaments: A Summary of Comparison Testing ......................... 36 


\section{List of Figures}

$\begin{array}{lll}\text { Figure } & \text { Page }\end{array}$

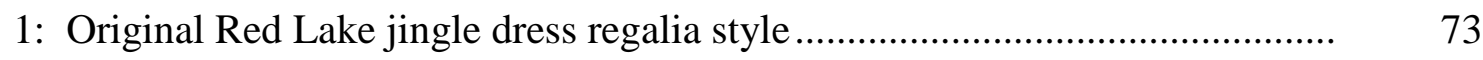

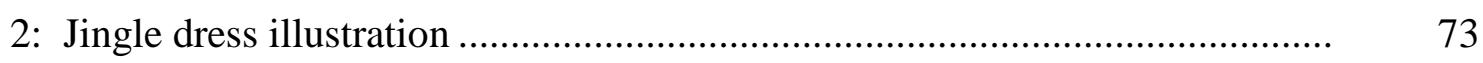

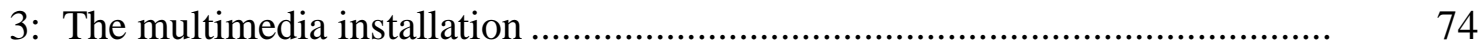

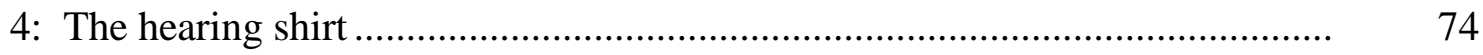

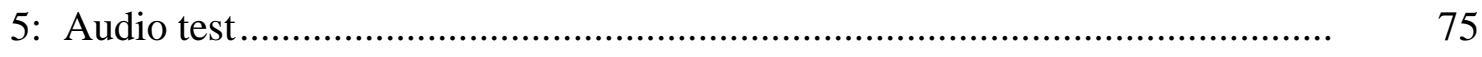

6: Speaker coil construction and materials consideration tests......................... 76

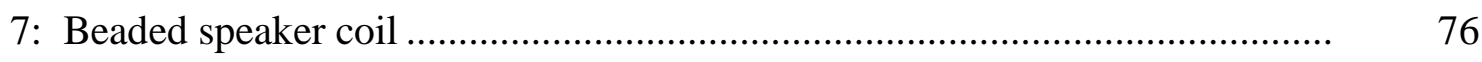

8: Customized PCB with amplifier .............................................................. 77

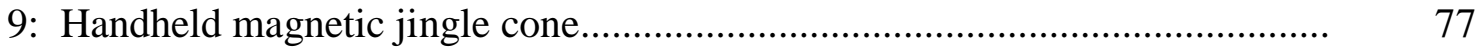




\section{CHAPTER 1: INTRODUCTION}

Canadian museums are important institutions; they are establishments for the preservation of material culture and serve as key agencies of research and education. In Preserving What is Valued, Miriam Clavir (2002) defines museums as non-profit, permanent institutions that serve society and public interests (p. 47) by acquiring, studying, and preserving artefacts that are esteemed to have intrinsic value through links with important contextual implications or associations. In this environment, tangible and intangible elements can be interpreted and shared with larger audiences; museums are a forum for critical thinking, experience, and even entertainment. Through curated exhibitions, museums strive to offer visitors both a first-hand glimpse and a deeper understanding about an artefact's cultural and historical significance. Clavir reminds us "in Western society there is an increasing recognition of diversity and particular minority 'stakeholders' whose special needs must also be served” ( $p$. 47). Adapting the built environment in museums is one way to serve special needs of visitors. However, other highly complex visitor interactions and experiences must also be assessed to establish new paradigms for inclusion and accessibility.

\subsection{Museum Policy for Restriction and Access to Physical Interactions}

Many museums favour a "look but don't touch" artefact policy that is primarily regulated by a visitor's own self discipline. To further ensure that human interaction will not impact the survival of a fragile artefact, environmental barriers such as glass display cases and low-lighting are often used in museum exhibits. Items that are too fragile to be displayed may need to be stored in special facilities. For example, clothing artefacts could be stored flat in darkened drawers to further restrict human experience and interaction. In certain instances, museums must also follow special health and safety measures, imposing physical barriers that protect humans 
against artefacts that exhibit toxicity. Certain First Nations sacred items require another type of spiritual protection, such as not being seen or touched by members of the opposite sex. This could mean that a toxic or sacred artefact might not only be restricted from public exhibition, but it could also be off-limits to curators and conservators who desire access for study purposes.

Replicas are often used to mediate experiences which would not otherwise be accessible to visitors. For example, an original artefact may not be physically robust enough to exhibit or may have been removed for conservation or study purposes. In this case, a replica or media representation is often used in place of the original to stimulate visitor engagement. Through the use of a replica, touch, manipulation, and interaction permit visitors to gain a better understanding. However, the capacity of a replica to generate compensatory experiences and entertainment visitors still cannot match the perceived value of experience with an original piece. This form of "experience mediation" calls authenticity of the experience into question. Meditative interactions in exhibitions must be further addressed by curators if the replicas that prolong the life of cultural property also impede a visitor's ability to directly experience the bona fide artefact.

\subsection{Research Aims of this Study}

Barriers profoundly impact a visitor's direct and authentic experience in the museum. Beyond the built environment, a number of other barriers serve to further confound these very institutions in providing experiences to the public at large in an inclusive manner. Museums are conflicted between following strict guidelines to protect cultural property and catering to the diverse needs of visitors and cultural groups. Hence, this study looked at how a fashion museum could curate inclusive, accessible experiences with artefacts through the implementation of 
interactive digital technology. With so many factors to evaluate, the scope of this study is limited to a particular focus in asking the following three research questions:

1. Which visitor special needs could be better accommodated for in the museum of fashion?

2. How can museums of fashion employ digital technology to curate inclusive, accessible experiences with artefacts?

3. How can digital technology be used to ensure that a clothing artefact will not be misrepresented through the curatorial process in a fashion museum?

\subsection{Planning for the Accessibility Mandate for Ontarians with Disabilities}

Recent mandates for accessibility and inclusion for more than three and a half million citizens living with disabilities (Statistics Canada, 2012) challenge Canadian museums to assess current practices, services, protocol, and infrastructure. Many aspects of these mandates focus only on improving services and modifying the built environment in public institutions to accommodate for people with visual disabilities. In Canada, the Accessibility for Ontarians with Disabilities Act, 2005 (AODA) requires public institutions to develop and implement accessibility standards with "respect to goods, services, facilities, accommodation, employment, buildings, structures and premises on or before January 1, 2025" and provide "for the involvement of persons with disabilities" (Ministry of Economic Development, Employment and Infrastructure, 2009, c. 11, s. 1). Focus on the visual experience of culture in museums means that the 836,000 Canadians who have self-identified as having a visual disability are being underserved by these public institutions. Furthermore, the National Coalition for Vision Health (2011) expects that within the next 25 years, the population of visually impaired and blind Canadians will double.

An appropriate next step would then be to examine other barriers in public institutions 
that are not being fully addressed by these mandates. For example, sensory and perceptual barriers in the mainly visual experience of exhibitions clearly need to be examined in the museum setting so they can cater inclusively to visitors with visual disabilities. Many cultural groups within the First Nations also express a need to honour religious beliefs, heritage, and treaties through hands-on interaction, and ritual use of ancestral items that are normally housed in museum exhibitions or stored collections. In both circumstances, sensory restrictions on touch and vision interactions limit the experience, and only provide an incomplete perception of the artefact. First Nations groups and museums have recently teamed up in a number of cases concerning providing access to cultural property. Although the issue of ownership of cultural property is far from being settled, a relationship with First Nations communities has led to the reworking and decolonisation of curatorial practices and preservation policies in a number of Canadian museums. For this reason, it stands to reason that elements of a First Nations approach, combined with sensitivity for the special needs of people with visual disabilities, could also be effective in the development of a more inclusive museum experience.

\subsection{A First Nations Approach to Cultural Preservation through Interaction}

The First Nations challenge many established hegemonic notions of artefact curation through their approach to preservation in museums. Primarily, the relationship between people and artefacts is paramount. Cultural preservation happens first through people and their personal recollections. Clavir (2002) defines this relationship as the principle driving force behind effective cultural preservation. Secondly, preservation of material culture is promoted through its [ritual] use. In this context, each artefact serves its purpose in the traditions in which people participate. It is customary to "feast" a sacred First Nations item like a hand drum or rattle. This means that, like a living being, it is ceremonially offered food from a sacred feast. It must also 
express its sound to fulfill its purpose, and be cared for in a way that is not catered to in Western museums. Even if the sacred item is fully intact and considered to be an artefact of value by Western perspectives, it could be viewed by First Nations groups as "lifeless" (Clavir, 2002). In the latter perspective, the sacred item cannot continue to fulfill its intended purpose, therefore preservation efforts should be ceased and arrangements for laying it to rest should follow. Furthermore, holding the wishes of the community in high regard is another invaluable factor which forms the First Nations perspective on the curation and preservation of cultural property.

In order to better understand and promote inclusive, accessible, and cultural experience in the museum of fashion, this study relied upon a number of creative activities that were driven by a practice-led methodology and was influenced by a First Nations approach. It explores traditionally accepted limitations of the Western museum visit and evaluates auditory modes of engagement with a cultural artefact. In particular, the research focuses on contributing to the experience of an artefact through interactions with sound, alternate sensory stimuli that is often overlooked in fashion museums. The unmediated sensory experience of the cultural artefact was inspired by the exciting, but largely untapped potential of "wearable sound" interactions that were made possible through electronic-integrated clothing. The Jingle Dress designed for this study serves both as a cultural, material, and visual artefact as well as an interactive instrument of sound. The technology embedded within it further embodies tangible knowledge generated through the artist's process. Furthermore, a multimedia installation plays a fundamental role in creating a context for generating authentic visitor-artefact interactions. The installation augments both the visual attributes and the "living" sound of the garment, thus emphasising modes of perceiving fashion that go beyond the artefact's material qualities. The artist's creative practice 
sheds new light on the current situation in fashion museums and provides grounds for recommendations for the endorsement of accessibility in public institutions of culture. 


\section{CHAPTER 2: LITERATURE REVIEW}

This literature review on museum environments covers four key areas: (a) curatorial challenges, (b) experience, (c) technology-enabled exhibitions, and (d) interactive sound installations. Barriers to interaction will be identified and discussed in context with museology and current curatorial practices. This section provides a background to the problem of forbidden interaction with museum artefacts, identifies compensatory efforts that are being implemented, and justifies why alternative solutions should be considered. Next, the role of sensory perception is contextualised with both art and technology to provide a standpoint for defining "experience." The literature review then segues to propose that through the elimination of mediatory devices, experiences have greater potential for inclusivity and accessibility. In other words, greater understanding and the synthesis of deeper meaning can be generated through "more potent" direct interactions as opposed to indirect ones. Focus then shifts to recent museum initiatives that are geared toward providing accessibility in "technology-enabled exhibitions." Several different types of digital media that aid in the promotion of interactive museum visits are discussed; certain are specific to providing equal opportunity for members of disability whereas others are geared toward the general public. The review also reports on creative practices that strategically relate electronic media and wearable artefacts to create interactional experiences within an exhibition setting. The final section of the literature review looks into recent artistic practices where sound is employed in an interactive installation.

\section{1 Curatorial Challenges}

Textiles present expensive curatorial challenges. Exhibitions can be costly due to bespoke displays, but special measures are also needed for storage and preservation. This is because textiles deteriorate in the mere presence of oxygen, fungus, oils from human touch, physical 
pressure, and even the force of gravity over time (Palmer, 2008, p. 36). Whether on mannequins or lying flat in a drawer, garments are often placed into controlled environments in order to prolong their longevity. In Exhibitions in Museums, Michael Belcher (1991) points out that museum display cases are specially designed to protect artefacts against theft, light exposure, pollutants, dust, changing humidity levels, and insects (p. 122). For example, sunlight and manipulation would quickly disintegrate the Royal Ontario Museum's collection of Queen Alexandra's weighted silk gowns. The gowns are especially fragile because the metallic salts used in the $19^{\text {th }}$ century to add weight to the fabric during the manufacturing process also greatly hasten the destruction of silk fibres. In other cases, special storage for an artefact may be required to protect humans against potential health and safety risks. Pesticide-treated aboriginal ceremonial blankets at the Canadian Museum of History in Ottawa and $18^{\text {th }}$ century mercurycontaminated felt hats at the Victoria and Albert Museum have been placed in restricted access storage containers to prevent staff from being exposed to dangerous toxic compounds.

To the chagrin of many visitors, museums continue to favour the policy of encasing artefacts in protective environments. Belcher (1991) investigates exhibition practice by addressing design considerations such as improvements to display cases and lighting, which promotes both preservation and effective communication between a museum and its public, but he does little to dissolve the current strict limitations on interaction with artefacts. Amanda Wade (2007) argues that the display case has become a restrictive sensory barrier that prevents a museum's public from attaining a complete learning experience (p. 23). Joanne Entwistle and Elizabeth Wilson (1998) explain that vital sensory cues that can only be generated by movement and direct interaction with a clothing artefact are compromised in typical museum exhibition: 
The costume museum makes the garment into a fetish, it tells us of how the garment was made, the techniques of stitching, embroidery and decoration used as well as the historical era in which it was once worn. What it cannot tell us is how the garment was worn, how the garment moved when on a body, what it sounded like when it moved and how it felt to the wearer. Without a body, dress lacks fullness and movement; it is incomplete. (p. 117)

Visitors naturally find ways to cope with limited sensory access to artefacts in a fashion museum display. Certain features of a garment, such as its interior finishing or back view, might be hidden in an exhibition. Environmental lighting might also obscure visual acuity. Without adequate opportunity to gather visual information one must rely on cues from his or her memory (Palmer, 2008, p. 32) to gain a deeper understanding about a clothing artefact. If a person cannot see a garment, and has never "experienced" the likeness of the material's particular texture through touch, sound, or smell, how can he or she judge if their perceptions are accurate? Moreover, if memory is used as a tool to evoke the intrinsic sensations of a textile or a garment, is the resulting "experience" of it only a figment of the imagination?

Curators might decide to construct physical copies of the original; replicas that will be used to demonstrate important characteristics that resemble the original. When she was unable to source an original, Diana Vreeland (1974) was notorious for producing replicas of artefacts for dress exhibitions such as Romantic and Glamorous Hollywood Design. Rick Hill (2014), an aboriginal researcher who works as an Indigenous program director the Smithsonian, has integrated replicas of museum artefacts as part of his lectures. He recognises, especially in aboriginal youth, the powerful emotional connections made when holding a Wampum belt replica representing the original Wampum historical treaties of their ancestors. A "hands-on 
experience" with materials and textile manufacturing equipment is also central to interactive learning at the Textile Museum of Canada.

Despite these efforts, reproductions do not "move" visitors; copies are perceived as lacking authenticity and commonplace. For this reason, the ability of a copy to channel the value of an emotionally compelling experience is less than it would be with the real artefact. An analogous argument would be in comparing a well-made knock-off and designer original; the copy is perceived to lack quality, value, and status. As such, this study will begin to explore ways to promote compelling experiences with real artefacts through accessible sensory interactions while maintaining a reduced risk of their endangerment.

\subsection{Experience}

What is experience? Merriam-Webster (2014) outlines no fewer than five definitions for this word; many of them are relevant to the various components of this study. A principle definition requires "direct observation of or participation in events as a basis of knowledge." Other dictionary entries situate experience as an affective state that can only be personally achieved through direct participation or observation. It assumes that time was spent in a particular situation and that a certain personal knowledge has resulted. Ironically, the concept of experience can also transcend direct personal knowledge if the situational knowledge can be attributed to the "conscious past of a community or nation or humankind generally." In any case, the ways in which an individual can directly observe and participate must be contextualised to better weigh the impact of any outcomes.

The scientific study of how our senses capture information is the pretext to understanding the essence of experience. Frank Geldard's (1972) The Human Senses describes physiological tissues and structures that receive and transmit messages from stimuli in our environment as 
sensations are interpreted. He maps out the range of limitations, or physical capacity, of the sensory perception process in human subjects. This is useful in determining which effects will be perceived in sensory receptors of the brain when they are targeted by a stimulus. Unfortunately, none of the empirical data gathered bridges over to the cognitive science of perception. None of the perceptual implications of people with diverse sensory abilities, for example partial vision or hearing, are considered in Geldard's discussions either. Understandably, it would be difficult to recommend how to arrange sensory stimuli to generate a single perception of an experience when an infinite number of variations in anatomies and abilities exist.

Looking beyond the measurements derived from the physical science of sensory perception, the museum experience can also be measured through a visitor's emotional reactions. Belcher (1991) defines exhibitions as having the ability to intentionally elicit both sensory and emotional reactions (pp. 58-66). John Dewey (1958) takes an in-depth look at this phenomenon in Art as Experience. He outlines boundaries to "having an experience": structure, perception, and temporality. Dewey defines which important role these interactions play in sorting out the meaning from an experience (p. 35). His philosophical views on the interrelations that form between humans and an expressive object, and in what capacity humans contribute to the relationship, demonstrates the intangible value of an experience. This work helps one to understand the relevance of interactions and how they might be formulated to trigger sensory and emotional experiences during the study's creative component.

Close proximity to art, which in this context is an artefact of clothing, can place the observer at a focal point for an "historical" experience. In his article, "The Historical Museum," Johan Huizinga (1920) defines "historical sensation" as a particular aspect of the relationship a person has with art: "the feeling of an immediate contact with the past, a sensation as deep as the 
purest enjoyment of the Arts you touch the essence of things, the experience of Truth through history" (p. 258). It is embodied by the intense, unsolicited sentiment that has been noted to happen as an observer engages a work of art. The "touch" described does not mean literally touching an artefact; rather, it depicts an emotional reaction to observation. This suggests that perception, through sight, hearing, smell, and even proximity to an artefact, plays a heightened role in the power of experience. The experience of an historical sensation is further likened to the sensation of hearing the past rather than seeing it, which places it in the category of immersive experiences (Benschop, 2007, p. 186). If hearing sound in an immersive environment can contribute to a visitor's experience of the past, then it can certainly be considered as an appropriate tool for bridging the gap of cultural accessibility in institutions where visual barriers exist.

Many experiences in the realm of digital technology are reliant on Human-Computer Interaction (HCI). John McCarthy and Peter Wright (2004) cover a plethora of HCI experiences that have proliferated in the past decade with the evolution of mobile technology. Human emotion derived from interactions with technology may also be analysed through numerous qualitative means. McCarthy and Wright propose six processes for interpreting an HCI experience: anticipating, connecting, interpreting, reflecting, appropriating, and recounting (pp. 124-127). These processes share certain similarities with human-based learning, communication, and storytelling. If the importance of analysing a HCI experience is to define how immersive or fulfilling the individual participating in the interaction actually perceives it to be, then it could be used to assess the visitor experience generated by a narrative of inclusive interactions in a multimedia installation. 
While a person with low or impaired vision could be considered as having a major disadvantage when encountering a visual artefact, he or she could achieve a more meaningful sensory experience through $\mathrm{HCI}$ in the form of adaptive wearable electronic technology. It is important to differentiate that the technologies to be discussed here mediate experience since they "translate" rather than "simulate" sensory data; rationale for a non-mediated experience will be discussed later. The VOICE VR is one example of software technology that is used in tandem with a wearable apparatus for blind people to experience vision. It detects objects in the wearer's environment and performs a "live" translation of the digital data into sounds (Doward, 2014). After a period of neurological adaptation, the brain can arrange sound signals from the device into a type of "synthetic" vision. Dr. Paul Bach y Rita has also worked with neuroplasticity and pioneered sensory substitution devices. For example, his Brainport device helps the user perceive sight, or rather perceive shapes, after several hours of adaptation. Electrical pulses from alternate sensory data, such as the taste, fool the brain into interpreting the environment in a new way (Collins, 2007).

The Haptex virtual reality (VR) glove research project conducted by Miralab, concluded in 2007 with the creation of a system that could digitally replicate human touch interaction with fabric. Some of Haptex's goals were to produce live interaction through complex electronic and software systems to enable the remote haptic manipulation of fabric over distances. Unfortunately, this technology focuses on simulating the movement of a virtual fabric in response to touch, rather than reproducing the texture effects of a fabric through digital means (Miralab, 2007). Understanding how the brain compensates for sensorial limitations such as visual impairment is an extremely valid point, but the HCI devices discussed here focus chiefly 
on sensory substitutions. This study differs in that it concentrates on using digital technology to supplement additional stimuli rather than on bypassing a usual mode of perception.

There are various other points concerning new substitutive device technology when considering quick and simple implementation in museums. For example, the VOICE VR software and apparatus, Brainport, and Haptex VR glove are still in preliminary testing phases and have not yet been ethically or commercially approved. If they were to be approved in the near future, the cost and time frame required for a blind person to fully adapt to and benefit from this technology would become barriers in themselves. Tailoring a sensory substitution device for individual anatomies and abilities means that their use could not yet be endorsed on a grand scale for use in museums. Another point for consideration is that these types of technology might facilitate a blind person's integration into society, but it certainly would not necessarily help society adapt to their own needs. Moreover, investment in the research and development of sensory substitution devices could divert resources, impeding the advancement of urgently needed strategies for museum accessibility and inclusive visitor experiences.

\subsection{Technology-Enabled Exhibitions}

While artefact preservation is a major concern for museums, accountability in satisfying patrons' mainstream needs and special needs must be considered from a technological perspective. Since touch and vision play large roles in experience, museums allocate large budgets for interactive media in the hopes that it that will compensate sufficiently for exhibition components that are inaccessible to people with disabilities. Audio loops for hearing aid users and touch tours or exhibition interpreters for people with visual impairment are some of the more common resources that are available in many museums. A growing trend reveals that more museums are engaging visitors with interactive, media-based, complementary learning aids as 
well. Museums are acting on a growing public demand for spectacle that is slowly overtaking public demand for information (Steele, 2008, p. 11). The resulting institutional shift toward both education and entertainment has been popularised by the term "edutainment" (Stoll, 1999). Museums must now also follow inclusive initiatives to provide barrier-free environments and services in these interactive "edutainment" strategies (Fitzhenry, 2013). While interactive components or facilities and services upgrades create additional costs, these upgrades translate into increased profitability for museums and sponsors as their clientele base increases.

Developments in technology have increased the complexity of interactions can that occur during a museum visit. Amanda Wade (2007) differentiates exhibition interactions into two categories: "low level interaction, opening a drawer and flipping a panel, to high level interaction, trying on clothing and using computers" (p. 2). Other aspects of a museum interaction include the type and level of participation required from visitors. Nina Simon (2010) explains how social objects in the museum promote valuable "teaming up" where co-operation and participatory interactions are needed for museum experiences to reach full potency.

Initiatives using digital technology to stimulate entertaining and educational interactions with artefacts in the fashion museum have risen in popularity in the last several years. At the Montreal Fine Arts Museum in 2011, Jean-Paul Gaultier's exhibition From the Sidewalk to the Catwalk captured the curiosity of couture-hungry visitors with its innovative technological interactivity. UBU, a local theatre company, animated display mannequins with mechanics and digital audiovisual projections, giving them expressive faces, voices, and the ability to spontaneously move (Rabinovitch, 2011). Later in 2013, the Art Gallery of Ontario exhibition, David Bowie Is, featured video clips and a mobile RFID audio "guidePORT” system by Sennheiser. The interactive elements in the environment were formulated to "recreate the 
atmosphere of a live performance" (Grimmer, 2013 [Video file]). The audio guide headset, equipped with a RFID reader, would activate different recordings according to a visitor's proximity to RFID tags dispersed throughout the exhibition to create a $3 \mathrm{D}$ immersive audiovisual experience. As visitors approached a specific artefact, music and a short narration related to the artefact would "fade in" and then "fade out" as they distanced themselves from it.

Initiatives to create participatory museum interactions are also quickly expanding into the virtual realm. Since 2012, the Social Fabric app has been available to visitors as a digital learning initiative at the Textile Museum of Canada (2010). By using their smart phone to scan a QR code located in captions beside a museum artefact, visitors can instantly access additional information and post their own comments on the museum website. Nancy Proctor (2009) also focuses on the "virtual" museum experience. In her roles as former Head of Mobile Strategies and Initiatives at the Smithsonian, Co-founder of the Museums and Mobile organization, Digital Editor of Curator: The Museum Journal, and current Director for Digital Experience at the Baltimore Museum of Art, Proctor uses web-based digital technologies such as mobile apps that function with QR Codes, touch-screens, and virtual online tours. Proctor's new mobile architecture focuses on three main directives for managing mobile content and museum services, especially for learning about artefacts. These are: offering the curator's general and detailed understanding, giving a visitor access to alternate hypotheses, and promoting related functions such as messaging, commenting, surveys, emailing, "favouriting," photos, et cetera (Proctor, 2009).

While it would be difficult to contest the benefits of integrating digitally supported modes of interaction into the contemporary museum experience, it may still fall short in several major areas pertaining to inclusive accessibility. For a person with partial vision, complementary visual 
animations and projections may be difficult to perceive, and may even skew his or her vision of the main artefact. RFID audio-tours may make the tour more eventful, but the seeming randomness of audio "zoning" could make unassisted navigation problematic for many individuals. Interactive learning tools, like the popular touch screen, may also pose difficulty for people with visual disabilities in museums. Not only can smooth user interfaces not generate Braille text for blind readers, but they may lack easy functions for people with low-vision to quickly change text size or contrast levels. Additionally, young children, elderly persons, or people with low mobility may find it difficult to operate interactive displays that require complex gestures or a high level of hand-eye coordination. Other accessibility complaints might hinge on a visitor's loss of independence when guides must accompany people facing language barriers, analphabetic individuals, or people with mental different levels of acuity.

The previous list summarises a number of common oversights in considering inclusion within the technology-enabled exhibition. The review of contemporary fashion exhibition technology also provides a basis for comparing visitor interactions according to direct versus indirect and mediated versus non-mediated in designing media-enabled experiences that are accessible for all audiences. However, a more fundamental oversight would be in the lack of prioritisation of actual relationships formed through interactions with an artefact. It's important to avoid the trap of shifting importance away from the relationship between the artefact and the visitor by using technological platforms to mediate their experience. There is certainly a danger that visitors will get distracted by, and only remember, the complementary digital media interactions rather than the value of the genuine artefact. There is also concern that museums are moving into a virtual realm, serving a target "client," rather than the individuals or communities who value real interactions with an artefact. This offers convincing insight for the need to create 
co-operation and participatory interaction that relates people, non-mediated artefacts of fashion, and experience in the museum setting.

\subsection{Interactive Sound Installations}

Sound sculptures have paved the way for the non-mediated experience of sound art and sound interactions in the modern museum. In June 2013, the Sojourn exhibition at the Denver Art Museum featured sculptural "Soundsuits” by artist Nick Cave. Created originally in 2004, these sculptures divert a viewer's attention to the audio dimension through dance, shaggy materials in motion when performed live (Denver Art Museum 2013). Unfortunately, when displayed in a static museum exhibition, the audio experience of these costumes is nullified. While immobile displays in museums lose their kinetic 'living' sound, interaction with electronic media installations can reproduce the sound of an artefact in limitless ways. For example, kinetic sculpture and sound artist, Marla Hlady in her 2008 installation Playing Piano deconstructs a player piano to show elements of that would normally be hidden from view. The distortion of time, experienced through a manipulated melody, allows us to study these components within an alternate context (Hlady, 2008). Peter Vogel's (2006) Interactive Sonic \& Light Sculptures is an example of sound generated by direct visitor interaction with musical structures. Visitorimprovised shadow play over photocell sensors and microphones in the sculptures triggers seemingly random electronic compositions (Vogel, 2006). While these pioneers clearly demonstrate that simple electronics can create interactive audio accompaniment for visitor engagement, the integration of these systems in the fashion museum is pending conclusive solutions to material conservation.

In the Digital Age, new forms of media and ubiquitous computing are being used to augment the experience of clothing. An increasing amount of technological fashion products are 
now available on the market. However, since the 2007 commercial release of the LilyPad Arduino (Buechley, 2007) popularity of "soft circuitry" is on the rise. Academic research and doit-yourself (DIY) online tutorials have inspired enthusiasts, on their own or in small tech clusters, to explore wearable audio and new sensory experiences through sound. The Thumping Threads Vest (Thumping Threads Team Members, 2009) and the Flutter Dress (Correll, 2012) are both vibro-tactile fashion developments that use audio to augment the experience of people with hearing impairments. This is a more passive, or reactive, type of interaction because the garment's response is a feature "programmed" to provide a specific experience. A vibro-sonic approach to sound does produce adequate ambient sound and is less appropriate for this study because it offers a more "prosthetic" type of sensory substitution which is intended as compensation for a hearing disability. Not only does a vibro-tactile garment mediate the wearer's visual experience, but the range of sensory effects produced is also extremely low.

SHOWstudios' (2006a, 2006b) The Sound of Clothes installations Synaesthesia and Anechoic involve the sound of fashion garments, which are digitally recorded and manipulated. Digital media helps free sounds from the chronological context, focusing on the experiential sensation of the acoustics produced by a garment. Alyce Santoro's (2012) Sonic Fabrics are woven with audio cassette tape, and play sound when a tape-head reader is dragged over sections of the fabric. The Accouphène Tuxedo is embroidered with conductive thread coils. Magnetic induction passed over the coils produces high-frequency squeals (Leclerc, 2006). These are examples of truly direct, non-mediated interactions in wearable sound technology because each person would experience a unique element of the garment's sound depending on how they manipulate materials and technology. The perception of an experience can be highly variable, especially because it is difficult to reproduce exactly the same sound twice. Thus, in engaging 
physical attributes of clothing artefacts, these installations indirectly shed new light on the current situation of museum challenges and limitations for the promotion of creative curatorial practices. 


\section{CHAPTER 3: METHODOLOGY AND METHODS}

This creative study was comprised of a number of research methods within the scope of an overlying process to explore gaps to inclusive accessibility in the museum of fashion. These methods were instrumental to the process in that each would become relevant directing the order and aims of ensuing research activities. Both a practice-led artistic process and aboriginal spiritual knowledge (Lavallée, 2009, p. 22) guided the framework for the iterative development of content and fueled the synthesis of a multimedia installation. Networking and community interaction also played influential roles in the contemplation of research questions and planning a logical progression of content creation. From these interactions, the artist was inspired to make her own Jingle Dress as a tool to examine the dilemma of accessibility and the representation of cultural artefacts in fashion exhibitions. Subsequently, an interactive sound installation that was built around the exhibition of the Jingle Dress presented visitors with additional experiences of artistic expression and cultural knowledge. Within the exaggerated sensory context of the installation, barriers to experience in fashion museum environment could be better simulated. Observations of how community groups participated, of how expert consultants contributed, and of how visitor interacted with wearable technology provided the artist with ample results to evaluate and interpret. Diverse media were used throughout the study to document the evolution of the artefact and to capture how it might be perceived by a visitor in the artistic installation.

\subsection{Practice-Led, Indigenous Artistic Research Process}

The artist initially approached this research as a conceptual exploration of inclusion and accessibility in the fashion museum. Yet it was prior experiences as a fashion museum visitor, as a retail shopper, and as a fashion designer that influenced the artist to begin with a systematic product design-type schema. In this light, commercial expectations such as design appeal, 
marketability, quality, cost, and production timeline rose to the forefront and began to steer the process. At that junction, the research of niche markets in relation to fashion museum curatorial challenges revealed a number of unexpected factors, all requiring deeper consideration and a more thoughtful approach. These conceptual connections were maintained but methodologies were further expanded to encompass artistic and experiential associations with cultural artefacts such as: sensorial, emotional, and spiritual.

Unlike many fashion, design, or museum curatorial frameworks, which are typically based on commercial models, a practice-led methodology was ideal for the artistic freedom and flexibility required for generating experiential knowledge from this study. A practice-led approach is viewed by most artistic researchers as "an umbrella term indicating that the practice leads the researcher rather than being announced merely as the subject of it" (Arnold, 2012, p. 19). Graeme Sullivan (2006) notes that "what distinguishes this kind of research is the multiplicity of ways of encountering and representing experience" (p. 23), a factor that was extremely pertinent to this study. Although no single model for practice-led artistic praxis exists (Arnold, 2012, p. 19), it has become synonymous with the conscious process of inquiry that draws upon the experiential relationship of the artist to generate evocative knowledge through his or her active employment of materials to explore a research question. It assumes that one cannot yet assimilate knowledge about something without having experienced it first-hand (Borgdorff, 2011, p. 61; Millward, 2013, p. 132).

As a Métis person in the process of defining her identity, the artist was also required to situate and balance a personal relationship between Western and aboriginal principles that would guide her practice. Linda T. Smith's (2012) Decolonizing Methodologies: Research and Indigenous Peoples suggests ways in which indigenous methodologies can be used to "rethink" 
and "rework" research practices, institutional operations, and community involvement. For example, indigenous researchers may look to the unconscious and intangible when seeking knowledge thorough experiential relationships. Lavallée (2009) explains: "Indigenous research is not qualitative inquiry; however, the methods used may be qualitative" (p. 36). "Knowledge acquired through revelation, such as dreams, visions, and intuition, is sometimes regarded as spiritual knowledge, which is understood as coming from the spirit world and ancestors" (Lavallée, 2009, p. 22). Lavallée (2009) succinctly reasons for experience and emotion in the artist's indigenous lens on conducting research:

Indigenous research is not objective, nor does it see itself as unbiased. Following from the belief in interconnectedness, ... research cannot possibly be completely objective. Individuals conducting the research are necessarily connected to the individuals being researched, and all concerned are connected to all other living things. Emotions are connected to all mental processes. Every time we think, use reason, and figure, emotion is tied to that process; therefore, it is impossible to be free of emotion and subjectivity in research. (p. 23)

Artistic knowledge shares a common thread with spiritual knowledge in that it must ultimately "be acquired through sensory and emotional perception, precisely through artistic experience, from which it cannot be separated" (Klein, 2010, p. 6). Hence, this practice-led methodology can be said to focus on the contribution of qualitative knowledge that would come from the Métis artist's subjective reflections about the intersection of experiential factors arising at different stages, and from different sources, of the work in progress. In this way, importance lies with the intricacies of the process rather than on the end product. 
The fundamental elements of this practice-led artistic process were highly dependent upon the research setting, materials, tools, techniques, and documentation. The research setting would influence the artist's progress and conclusions in a very distinctive and subjective way (Mäkela, 2007, p. 160). Hence, she carefully considered the capacity of each material, tool, and technique to be used. An initial study of how these elements could be used individually or in a composition to explore the initial concept would thus determine the logic for research activities that would follow. Sullivan further defines the interplay between individual purpose and situational factors that arise within this type of artistic process as "transcognition," a process which is "iterative and strategic in nature as meaning is encompassed and negotiated" (Sullivan, 2001, p. 9). This study involved a vast degree of uncertainty and unforeseen challenges that were collectively vital to the artistic process and fostered the production of highly significant knowledge and potent artwork. Both the artefact and supporting documentation would later serve as evidence for the accumulation of experiential knowledge, and the importance of their roles in this study will be discussed later in this paper. Documentation included: an artist's workbook (with sketches, illustrations, patterns, and written ideas), observational photos and audio-video recordings for testing and process work, and a short video of the interactive installation.

\subsection{Regalia as a Symbol of Curatorial Struggle}

An unconventional Jingle Dress was chosen by the artist as a principal vehicle to explore accessible forms of experience in an artistic installation. This dress differs from typical female Ojibwe Jingle Dress regalia in that it was conceived with embedded sound technology, to solicit the experience of a cultural artefact through alternative modes of interaction and sensory perception. Tara Browner (2002) outlines the origins and context of healing associated with the Jingle Dress in Heartbeat of the People. Although prior to the arrival of Europeans, aboriginal 
women danced in sound-producing dresses adorned with shells, bones, teeth, or pieces of copper, Browner attributes the contemporary Jingle Dress to an Ojibwe father's spiritual vision in the early $20^{\text {th }}$ century. His vision resulted in the construction of a modest dress with rows of rolled snuff lid cones (Figure 1) and dance steps for his gravely ill, young daughter, Maggie White. As she danced, she was healed by these gifts to the Anishnaabeg people by the spiritual power evoked by the sound of jingle cones (Browner, 2002, pp. 53-54). The Jingle Dress is regarded by the Anishnaabeg of Ontario as regalia; it is a sacred, spiritual item of clothing that holds a narrative, meaning, and identity. They believe that dancing regalia "is not meant to be put under glass, like in a museum" (Belcourt, 2010, p. 21). When considering that a Jingle Dress would be rendered motionless in conventional museum conditions, thus impotent in producing a healing sound or tell its narrative, one can better understand the curatorial struggle to preserve cultural artefacts and the role of accessibility in the experience of them. The Jingle Dress was chosen for its symbolic role as a sacred, healing item with potential to impact on the well-being of social environments like the museum of fashion.

In order to proceed with making a Jingle Dress, the artist was confronted with many important decisions for design concept, and functionality. Since the 1920s the Jingle Dress has evolved into a popular type of regalia for female pow-wow dancers throughout North America (Crazy Crow Trading Post, 1997), however certain characteristics differentiate whether it is intended for spiritual healing or entertainment (Pheasant, 2006). Primarily, the Jingle Dress wearer must assume the role of responsibility that comes with being a healer and undertake spiritual learning about the sacred nature of the Jingle Dress while she constructs it. As a result she determined a need to consult Jingle Dress dancers, elders, and aboriginal centers in Toronto about specific requirements for this regalia. From these consultations, the artist determined a 
dress design that reflected her personal vision to become a healing Jingle Dress dancer. The artist represented her dandelion seed pod healing vision of the first through hand-drawn sketches. She then further refined it into a digital technical illustration (Figure 2) from which to develop a tangible, three-dimensional, cultural clothing artefact.

The dress pattern made by the artist included an A-line style skirt and bodice with sleeves and front zipper opening, fitted to her body size. The bust area was left unadorned for modesty. Furthermore, it was also constructed in a modest, single-hue, plain weave cotton, with humble embellishments and accessories. Wearable electronic technology for playing sound was embedded in a way to visually and audibly support the main style feature: 365 snuff lid cones suspended in rows from bias tape. The creation process also involved protocols such as giving an elder tobacco in exchange for specific Jingle Dress teachings, smudging self and materials with sage for purification, giving thanks for each material used, using materials resourcefully, working with good intentions, wearing cedar in any pockets present, and feasting the Jingle Dress as a sacred item before dancing in it.

\subsection{Interaction}

Both human-human and human-artefact interaction were considered within the context of this creative process. In developing an innovation, a researcher might "build a list of useful contacts on which to draw when it no longer seems promising to struggle alone with technological bottlenecks" (Pyka, 1997, p. 210). Human-human interaction defined the informal networking and external consultancy that were vital for understanding and developing technical aspects of the artefact. Human-artefact interaction addressed the contemplated design innovation in finding a way for visitors to physically experience the artefact while respecting parameters set by textiles conservation standards such as low-light and forbidden touch. Networking played a 
significant role in seeking specialised knowledge from community groups while hired consultants were indispensable for navigating technical issues with materials and digital tools.

Informal networking was instrumental in providing skills, knowledge, and personal motivation which influenced the artist's reflections about the research and creative practice of this study. Since she was not familiar with Ojibwe Jingle Dress teachings prior to this study, she sought to connect with a Jingle Dress Dance elder for advice. Although she did not succeed in connecting with an elder with this specific background, a plethora of information about the Jingle Dress Dance and aboriginal ways of knowing came through participation with the following organisations: Ryerson University Aboriginal Student Services (RASS), Native Women's Resource Center of Toronto (NWRCT), and Native Canadian Center of Toronto (NCCT). Supplementary teachings about aboriginal knowledge and experiences for the preservation of cultural artefacts came from a lecture at OCAD University about Wampum belts. Participation in an aboriginal graduate student retreat hosted by Queen's University Supporting Aboriginal Graduate Enhancement (SAGE) was fundamental in understanding current aboriginal views on the integration of technology and 'traditional' aboriginal practices. Other opportunities for valuable informal networking with electronics and sound experts were effectuated at: Toronto Wearable Meetups, InterAccess Electronic Media Arts Center, OCAD University, New Adventures in Sound Art (NAISA), Ryerson Laboratory for Interactive Fashion and Technology (RLIFT), Ryerson Engineering Hobby Electronics Club (HEC), Hacklab.to, and Ryerson New Media "hacknights." This was vital in gaining a working knowledge of electronics and experimenting with various components to building several wearable sound systems from scratch.

Consultants were hired for technical contributions to the creative component of this study 
where the artist's level of expertise was inferior. An electronic media specialist was hired to improve the volume, sound quality, and miniaturisation of the wearable speaker developed by the artist. An aboriginal beading expert was instrumental in teaching the artist various First Nations beading techniques and giving feedback on respectful protocol for certain aspects of the creative project and installation. A sound recording technician helped record the Jingle Dress in motion, master these recordings, and tweak output levels for effective playback from the fabric speaker. A metal sculptor was also hired to develop a large, hand-held stainless steel cone that was not included in the installation. Instead a smaller version with rare earth magnets inside a Jingle Cone was provided for visitors to amplify the acoustic resonance of the garment's fabric speakers.

The actual design for wearable speakers and sound system are adaptations of Hannah Perner-Wilson's (2011a, 2013) related models. Essentially, the artist committed to working with electromagnetic induction after experimentation with several types of sound actuators that are compatible with garments. Hannah Perner-Wilson's technique permits safe and easy adaptation to garments while producing a reasonable level of fidelity in sound produced by electric signals. However, the system did require special thought to conceive a feature that would permit a visitor to actuate the sound of the device without engaging in physical contact with the garment artefact it was embedded into. Electromagnetic induction was an appropriate method to initiate sound vibrations from a distance by moving a strong, rare earth magnet over the center of an electrified conductive thread coil. Perner-Wilson's "Sound Amplification Circuit" schematic for paper speakers found on A Kit of No Parts (KoNP) website was initially intended as a technological foundation for creating this type of sound system. However, the artist chose to pursue an integration of Perner-Wilson's mp3 player system in tandem with a "hacked" commercial 
speaker as seen in "Fabric Speakers" (Perner-Wilson, 2011a). The latter seemed more promising at the outset because it provided a more sustainable solution in terms of simplicity, longer battery life, and higher volume output. Subjective material testing, such as fabric rigidity thickness, fibre content, and effects from interfacing was also prescribed for determining effective sonic output and the effect of speaker materials. Stitching type, size, and tension as well as electrical resistance of various conductive thread types were also tested to determine four Ohm $(\Omega)$ resistance for an ideal speaker output. Other considerations dictated that the sound system components should be small enough for a seamless integration into the garment, yet easy to detach for garment laundering. The artist's criteria for the components to be "wearable" maintained that they should, in no instance, pose a threat to the safety of the wearer, to the garment itself, to visitors interacting with the technology, or to the museum displaying the artefact. A final concern was that the magnetic interaction should be low-risk and easy enough for any visitor to manipulate.

\subsection{Installation}

The multimedia installation (Figure 3) was creatively built around producing new, and highly variable, visitor experiences through sound. In a subdued visual environment, it presented an opportunity for visitors to interact with a cultural artefact without the use of touch, replicas, or other devices of sensory mediation. Above and beyond what has already been described for the functionality of the audio system embedded in the artefact, the artist also arranged particular aspects of the exhibition space to reduce physical barriers to access the location, and to build an immersive lighting and sound environment. Another aim was to observe how visitors would naturally interact with the installation. The observations of visitor reactions to the elements she 
had developed to engage inclusion and accessibility contributed to the body of experiential knowledge that would later influence the artist's recommendations.

To ensure visitor diversity, and to target a broad range of abilities, cultures and ages, invitations were sent out electronically to a number of groups and individuals. Ryerson University represents a diverse public; it is attended by approximately 40,300 students from 146 countries (Ryerson University, 2015), many of whom study in programs that engage inclusion and diversity for gender, ethnicity, sexual orientation, culture, and ability. Ryerson University School of Fashion undergraduates, graduates, and faculty were sent email notification via fashionnews@ryerson.ca that included a brief description of the artist's project, details of the free event, and a copy of the installation poster. The same information was forwarded in personal email correspondence and on social media to a number of other groups including: Ryerson Aboriginal Education Council (AEC), CNIB, Fibre Quarterly, Hacklab.to, Ryerson Creative Industries Program, Ryerson Hobby Electronics Club (HEC), Ryerson Aboriginal Student Services (RASS), Ryerson Department of Equity Diversity \& Inclusion, Ryerson Laboratory for Interactive Fashion Technology (RLIFT), Queen's University SAGE group, and Toronto Inclusive Design Meet-up Group. The installation was attended by approximately 50 visitors. Coordination of special accommodations, such as detailed directions or accompaniment to enter or exit the premises, was made upon request.

The installation was held in Toronto, Canada at the Ryerson University School of Fashion on Wednesday, April 2, 2014 from 4:00 p.m. to 7:00 p.m. To facilitate visitor access and an appropriate acoustic environment, the artist chose to set up the installation inside a small room located on the basement level of Kerr Hall West. A welcome table and wall poster situated in the adjacent stairwell was placed to intersect with human traffic both from visitors descending from 
the ground floor above and from visitors arriving by the southern ramped basement entrance. Visitors were greeted by the artist and a volunteer associate, encouraged to learn about and touch an interactive sample of the wearable speaker technology. Other "touch" items, a fully-beaded, spiral speaker medallion that will later be added to the Jingle Dress sleeve and a Jingle Cone, were made available at the welcome table for visitors to grasp an understanding of speaker anatomy and the interaction necessary to access sound from the textile. Here, the use of magnetic components was disclosed for pacemaker wearers.

The artist then accompanied small groups of visitors inside the eight by four foot room to experience the installation. Doors on both ends of the room were kept closed to isolate the installation setting from external noise pollution and a draped fabric backdrop on one end was in place to dampen echoes. Inside, UV-A lighting was used to showcase the white mannequin and Jingle Dress elements in the surrounding darkness. As the sound boards were placed on the floor directly behind the mannequin, their tiny green LEDs were a less distinguishable source of illumination. Visitors were encouraged to interact with each of the four 'active' wearable speakers and to discuss accessibility issues. In this way, the artist could observe and visitor interactions with wearable sound technology embedded in a cultural artefact of her design for later interpretations. The installation was documented through photography and video to show its kinetic and sound elements. 


\section{CHAPTER 4: RESEARCH PROCESS-A CHRONOLOGY}

The praxis of this study on accessible experience in the museum of fashion began with an investigation of garments, fabrics, and technology. Manipulating garments through touch or kinetic interactions to produce sound in the museum setting proved to be major conceptual hurdles. How could clothing artefacts be "hands on" and touchless at the same time? Hence, a number of prospective types of interaction to create sound through indirect touch were reviewed at length. The first, and most straightforward, option involved the use of a touch screen, which could be positioned within the periphery of the artefact. Although interactive, digital media display platforms can add to the overall entertainment and experience for many visitors, screens, by design, limit sensory experiences for people with visual impairments. Therefore, touch screens were ruled out as a viably inclusive solution. The second option required a wearable device to be directly embedded into the artefact. This type of adaptation creates limitless possibilities for design, functionality, and direct sensory engagement. In taking this idea further, many strategies were considered. Proximity sensors used to automate mechanical structures inside a garment was one of these strategies. The garment would change its shape in response to a visitor's physical presence, manipulating the garment's fabrics to make "noisy" sound effects.

Although this system clearly meets the criteria for inclusive and touch-less interaction, it directly opposes most museum preservation policies since the rapid destruction of the artefact through mechanical abrasion would be inevitable. Furthermore, if it were possible to reduce the abrasion by slowing the rate of kinetic manipulation, the sound effects produced by the "event" would also diminish, becoming far less obvious to visitors. Both of these options were dismissed because the artist judged that they would hinder a visitor in his or her participation and contribution to the interaction. Multi-sensory cues from the environment help generate spatial 
awareness and perception. Only in compiling these cues can visitors hope to elucidate a profound emotional experience and personal understanding of a particular artefact located within a specific environment.

\subsection{Wearable Audio Technology Research}

To better understand the scope of potential interactions that could be used in the context of this study, the artist set out by familiarising herself with wearable electronics. This included learning about basic electronic circuitry and textiles solutions for embedding or emitting light and sound. Instructional courses and workshops at InterAccess, an artist-run "public gallery, educational facility and production studio dedicated to the creative use of technology, electronic art and new media culture" (InterAccess, n.d., para. 1), and OCAD, a university that "strives to be one of the world's most significant specialised universities in the fields of art, design and media" (OCAD, 2015, para. 1). These two institutions offered hands-on workshops for creating personalised "wearables" with DIY electronic circuitry, techniques, and devices. These incorporated a number of different sensors, actuators, and "hacked" components. However, sound, noise, and music were rarely addressed in these courses. This is because sound requires highly specialised knowledge, such as physics, to fully understand the complex relationship between material properties and their effect on the propagation of acoustic sound energy (i.e.,

pressure waves) through time and space. Her aim in taking these courses was to achieve success with basic electronic exercises. Her positive results provided enough invaluable conceptual knowledge about light, sound, power, and resistance to inspire the practice of more complex trials.

New challenges involving waveform conversion and synthesis arose in the artist's next wearable-sound project called The Hearing Shirt (Armstrong, 2013). The shirt was designed to 
process live sound from a person's periphery and to convert its signal into real-time vibro-tactile emissions (Figure 4). Since sound is naturally analogue, the continuous waveform of sound captured by a microphone had to be converted by a microprocessor into discrete signals at a sufficiently high sampling rate, and compressed, to activate the vibro-motors. Furthermore, material properties of components and environment were impacting on the sound quality. The geometry of a space, and its surface of materials, can produce different of resonant effects such as sound muffling or echo. The orientation of speakers or microphones can also impact the characteristics of sound as it is emitted from a source or captured in a recording. Feedback from the vibro-motors was adding additional complications. The process of testing different configurations for wearable microphones, vibro-motors, and microprocessor program provided a great deal of insight on materials and environmental elements that needed to be refined for optimal sound quality in this study.

After that, several applications of invisible, yet engaging, methods of touch-less interaction were reviewed. The artist compared a number of platforms for this type of sensory interaction, including wireless sensor networks, radio-frequency identification (RFID) systems, and electromagnetic induction. Of these three platforms, electromagnetic induction with a conductive coil presented the most relevance. If applied in the context of museum exhibition, it would allow for the pre-recorded sound of a garment artefact to be reproduced directly by the display artefact through its embellishments. This was an important component in making the garment focal to the experience, which in this logic, meant that sound should concentrate a visitor's attention toward the artefact rather than toward peripheral tools or other environmental elements. It stood to reason that a touch-less interaction would entertain visitors and perhaps fascinate their interest in the science of electromagnetic technology because it is certainly 
uncommon for a garment to produce sound electronically. Furthermore, the coil itself would be a good choice for its smart simplicity; relatively easy to stitch into a garment, low cost, washable, flexible, and its conductive thread would add a pleasing decorative element. Schematics for two basic conductive coil audio systems were readily available online; however indications about coil materials and the results they would produce were not available. A diagram indicating favourable construction techniques and materials has been published online more recently (see: kobakant.at) but the artist has remarked several points where the results publish are misleading and inconsistent with her own speaker coil testing.

Preliminary testing was conducted to ensure that Hannah Perner-Wilson's theoretical model for constructing a fabric speaker could be replicated and made effective for the purpose of this study. Without knowing which factors could affect output, the artist set out to sew her first flat spiral air core inductor. The spiral was made on by sewing 0.15 centimeter cotton zigzag topstitching to secure silver core spun polyester thread onto muslin. Its diameter was 14.5 centimeters, with the silver thread spaced in rows approximately 0.2 centimeters apart. The speaker coil was then tested by trial and error with a rare earth magnet and an oscilloscope at the HEC (Figure 5) to determine limitation factors such as resistance, current, and frequency. Since no one present had executed this type of audio test before, the group was elated when an audible signal was finally achieved. Affirmations for audio frequency range, voltage draw, and fabric stiffness were then obtained from this high pitched "squeal."

At this junction, a variety of speaker coil samples were required for comparison. A set of ten coils in five different conductive filaments, and two separate diameters, were sewn onto a stiff interfaced nylon canvas (Figure 6). Filaments included: (a) 0.01 centimeter enameled copper wire, (b) 10-strand copper stereo cable wire, (c) Mettler Metrosene silver seven-ply core spun 
polyester thread, (d) 316L extra thick stainless steel six-ply multifilament thread, and (e)

Stainless steel 2-ply yarn. Coils were either nine centimeters or six centimeters in diameter and were made by zigzag stitching over the filaments placed approximately 0.15 centimeters apart. Each finished coil was weighed in terms of stitching ease, machine ease, and conductivity. Comparison testing results for each filament is summarised in Table 1. Further testing the performance of each speaker sample would have to wait for the development of a compatible audio system.

Table 1

Conductive Filaments: A Summary of Comparison Testing

\begin{tabular}{cccccc}
\hline No. & Description & Stitch ease & Machine ease & Conductance & Comments \\
\hline 1 & $\begin{array}{c}\text { Copper wire } \\
0.01 \mathrm{~cm}\end{array}$ & moderate & good & None & $\begin{array}{c}\text { Machine damages } \\
\text { filament }\end{array}$ \\
2 & $\begin{array}{c}\text { Copper 10- } \\
\text { strand wire }\end{array}$ & Difficult & poor & excellent & $\begin{array}{c}\text { Wire too thick, too } \\
\text { resilient }\end{array}$ \\
3 & $\begin{array}{c}\text { Silver core spun } \\
\text { thread }\end{array}$ & moderate & moderate & good & $\begin{array}{c}\text { High risk of needle } \\
\text { abrasion }\end{array}$ \\
& $\begin{array}{c}\text { Stainless 6-ply } \\
\text { thread } \\
4\end{array}$ & Easy & good & good & $\begin{array}{c}\text { Slow speed for best } \\
\text { tension }\end{array}$ \\
& $\begin{array}{c}\text { Stainless 2-ply } \\
\text { yarn }\end{array}$ & moderate & poor & moderate & Damages machine \\
\hline
\end{tabular}

In practice, problematic filaments were quickly ruled out. The most notable issues that arose during sewing were needle abrasion fraying on the silver thread and abrasion damage to the machine caused by the rough texture of the two-ply stainless steel yarn. As these issues occurred when threads passed through the needle, it was concluded that a cotton zigzag topstitch thread was the most desirable way to secure the conductive thread in place on the surface of the 
fabric. Even so, the thin, fragile copper filament was severed by topstitch pressure, resulting in non-conductance. The thicker copper wire had an extremely resilient structure, which made it difficult to regulate coil turns under the presser foot during machine sewing. Although it required a wider zigzag and slower speeds to maintain proper tension, extra-thick six-ply stainless steel thread proved to be an ideal choice. It was easy and quick to sew evenly spaced intervals. It would also not oxidize or lose conductivity though broken filaments with time. Furthermore, the coil demonstrated a tactile suppleness yet it maintained a tensile stiffness. The spiral "V" topstitch pattern over shiny, flattened metallic thread distinctly created an aesthetic dandelion clock look, which would later become a thematic element for selecting other garment materials and embellishment techniques.

Developing a wearable audio system to test the coil speaker samples ensued. For a number of beneficial reasons, the original plan for a wearable audio system was based on the KoNP "Sound Amplification Circuit" musical greeting card model. Primarily, the greeting card audio system is relatively simple, low-cost, lightweight, safe to operate on low-voltage, and are miniaturized enough to be considered "wearable." Secondly, some musical greeting cards are configured to record and play back customized of sounds or messages. This would also permit a quick, low-tech set up for testing a fabric speaker coil's viability. Consequently, the artist deconstructed a greeting card circuit and tested it first on an audio speaker then with the first speaker coil sample. Despite demonstrating positive results from an audio speaker, no audible signal was emitted when the original fabric speaker coil was hooked up to the greeting card system. Audible results were only achieved from the fabric coil speaker at a HEC meeting when a nine volt battery and TIP122 transistor were integrated into the greeting card system. This was 
a significant accomplishment, but unfortunately, its volume was not loud enough to provide an impressionable sensory experience.

It was evident at this stage that volume amplification strategies had to be investigated and secondary options entertained. Naturally, this first required an understanding of factors that contribute to volume in an audio system. Through consultation, the artist assessed that the lowtech, modified greeting card in Perner-Wilson's demonstrative system could not surpass certain limitations that had presented themselves during testing. Higher volume would require higher voltage, therefore low-voltage in the greeting card system meant that, with or without the TIP122 transistor, it could only deliver minimal sound amplification at best. Perner-Wilson's system operated a hacked miniature mp3 speaker device that was equipped with a small, yet potent, amplifier. However, the original miniature mp3 speaker employed in Perner-Wilson's audio system was no longer available on the market.

For that reason, the LilyPad "wearable" mp3 player was ordered for testing. Although setting it up proved to be tedious the artist was able to program the LilyPad to play mp3s from earphones. A major drawback of this device was the tall control knob; it not only created a height of nearly 3.5 centimeters, but without function icons, it was also problematic to manipulate. Additionally, volume settings regulated for standard earphone tolerances would not permit volume to be set high enough to be used with the fabric speakers. Electromagnetic inductance could potentially erase all of the data from the mini SD card. Looking back over the selection of audio systems, Perner-Wilson's example resurfaced as the best option for adequate volume when used in tandem with the simple conductive speaker coils.

Since trial and error would make synthesizing a customised audio system an extremely lengthy process, more consultation about electronic media was necessary. Improving volume 
could be achieved in similar electronic media by following online tutorials, but using components that differed from those used in the tutorials could easily skew outcomes. Moreover, the task of selecting components that were both compatible with each other and safe for humans to wear required a great deal of experiential knowledge in electronics and sound. Hence, an electronic media consultant was hired to develop an audio system with improved volume for the artist to embed into the design of her garment artefact. The project overview discussion between the artist and the consultant covered Perner-Wilson's schematics, coil speaker test results, ideal audio functionality, and illustration of placement on the Jingle Dress. The artist specified certain desirable "wearable" characteristics for the technology to be developed such as small size, mobility, simple interaction behaviours. An approximate timeline for deliverables, material requirements, costs, and several guidelines for the power functionality and safety of the audio system were also discussed. During the meeting, the consultant received all components and fabric speaker coil samples that had been developed by the artist. Over the next months, the consultant proceeded to develop a working amplification model that would be used for multiple speaker coils in the Jingle Dress.

\subsection{Research and Execution of Jingle Dress Regalia}

The artist was inspired to become a healer after a vision appeared to her 5 years ago, but she was slow to respond because it lacked context. In light of missing information, it was also unclear how to proceed and what form it should manifest itself. It was only in reflecting on a suitable cultural artefact to represent in her research installation that the context became evident. After considering numerous possibilities for clothing artefacts that lose their ability to produce sound in the museum setting, her interests honed in on a particular garment that had been previously overlooked: a Jingle Dress Dancer's Dress. The vision resurfaced during a spiritual 
consultation with a RASS Elder when vivid memories of this sacred item, honored at a recent ritual feast, urged her to pursue this direction. Thus, the artist's journey to becoming a healer through a powerful spiritual relationship with the Jingle Dress began with learning more about it.

Literature and various online resources were helpful, but interpersonal interactions with other members of the Toronto aboriginal community proved to be valuable to her research on a much grander scale. RASS staff and student Jingle Dress dancers were very forthcoming in passing along their personal knowledge. They encouraged contacting other local organisations to seek teachings from an Elder who would be more qualified to pass on specific spiritual teachings. The NCCT provided information by phone about classes for learning how to sew traditional regalia, bead accessories, and dance powwow styles. The NCCT program coordinator, a Jingle Dress Dancer herself, also addressed that being a Jingle Dress healer required commitment and responsibility, which is not a role to be taken lightly or frivolously. She recommended contacting the NWRCT for additional spiritual teachings support. On the contrary, the NWRCT receptionist who responded to the artist's email inquiry was hesitant and protective of sharing this knowledge. The receptionist replied by posing a number of questions, challenging the artist's personal vision and calling to become a Jingle Dress dancer. She also jumped to negative conclusions at the mention of including a Jingle Dress as part of an installation about accessibility in museums. Embedding technology into "traditional" regalia would be an aberration. In the emails, she referred to the artist as "not being native enough." The NWRCT representative said that she would not share this specific knowledge; sharing spiritual teachings with a non-indigenous person would be reckless on her part. This was a learning experience indeed!

The blatant evidence of exclusion was a reminder that accessibility issues present 
themselves on a number of levels in many institutions of culture, and only strengthened the artist's conviction. She wondered: if she had mentioned her Métis background in the inquiry, would she have received a different reply? This compelled her to contact the NWRCT director about the situation and she received an entirely sympathetic reply. The director, a blond blueeyed person who often had to defend her identity as person of indigenous heritage, made it clear that the receptionist had not replied in a way that reflected the organisation's mandate for cultural inclusivity. From that point onward, every effort was made by the NWRCT to provide contacts for spiritual teachings.

The next developmental stage involved translating the artist's vision into the material form of her Jingle Dress. While Jingle Dance resources hinted at typical silhouettes and styling trends, Regalia class outlined material requirements and construction techniques. For example, while some elements like short sleeves for summer heat were optional for the Jingle Dress, fringe styling and materials such as deerskin were more appropriate for the Women's Traditional Dress. Instead of using multiple eye-catching colours or flashy metallic fabrics as per contemporary pow wow styles, she aimed to use a sober, monochromatic hue that was typical of older Ojibwe Jingle Dress styles. Popular appliqués were avoided because the imagery would conflict with the modest symbolism of her dress design. While spirit animals or eagle feather appliqués may represent a person's identity or status, the dandelion was chosen for the dress's overall styling because of its symbolic resilience and potential for growth and change. Therefore, the main fabric colour of the dress was yellow-ochre stretch cotton sateen with white fake fur trimmings, yellow and white polka-dot cotton bias tape, and silver metal Jingle Cones.

The dress pattern was based on the artist's revised illustration. It includes long, bell shaped sleeves and an A-line skirt, both with multiple row of Jingle Cones in a spiral twist to 
evoke dandelion petals. The speaker coils were originally to be embedded directly on the chest, back, and bicep areas of the dress. However, due to technology development schedule delays they had to be transferred to a separate accessory yoke. This allowed for pattern drafting, muslin fitting adjustments, and final garment assembly to continue in the meanwhile. The rear zipper was moved to the font of the garment to facilitate donning the dress without assistance. The front, back, and sleeves were sewn together as entirely separate panels to permit more mobility in applying embellishments. To begin assembly, lengths of polka-dot cotton bias tape were prepared. Short strips were cut, knotted, and fed through a Jingle Cone. The top metal point of each Jingle Cone was then crushed with pliers to prevent it from slipping off the fabric tape. Lengths of bias tape were machine basted horizontally in rows to the dress according to the proportions of the dress illustration. The tape ends of each cone were pinned in place underneath the bias tape, which was then folded over and stitched in place. With a thin strip of imitation fur trimming between the layers, the top layer of bias tape was then folded over and topstitched. Assembling the major seams followed in sequence: shoulder seams of front and back, armhole, underarm-side seam, neckline facing, cuff facing, and blind hem stitching. After the embellishment steps were completed, the bulk of approximately 340 cones proved to be cumbersome in sewing and their weight resulted in a looser overall fit in the stretchy fabric, especially at the waist level.

Since the speaker coils were now to be embedded in a separate accessory piece, the artist started ideations for the decorative features of a neck yoke and knee-high leggings. These Jingle Dress accessories are customarily embellished with appliqués and beaded motifs that reflect other thematic elements of the dancer's dress. Although early beading designs, which featured depictions of the different stages of the dandelion's lifecycle, were eventually ruled out because 
they were too literal. Instead, she focused on only using beading on the speaker coil to highlight symbolic relationships in a less obvious way. The beaded design resembled a dandelion clock, effectively masking the technological and functional elements of the speaker. With a mirror in centre to protect against bad intentions, the underlying conductive thread spirals outward like an umbilical cord. This represents the interconnectivity of individuals and larger 'guardian' institutions who must participate with intentions from the heart to pass on the experience of a valuable source of knowledge.

Next, the patterns, muslins, and materials for the yoke and leggings were executed. Several layers were required for construction of the final pieces: cow suede, suiting canvas interlining, felt, and cotton facing fabric. The trimmings were identical to the Jingle Dress but the suede and cotton facing fabric were darker burnt ochre. Polka-dot bias tape was used as general edge finishing while the bottom yoke edge also had white imitation fur and 30 Jingle Cones. A zipper at the left shoulder made the neck opening of the yoke accessible. The leggings did not include imitation fur or cones at the edges and were simply laced in the rear. Finalisation of the yoke and leggings was then put on hold until the electronic component tests could confirm speaker material combinations.

Focus then shifted to testing speaker physical properties. Since the original fabric speaker coil test on interfaced polyester drill had revealed the need to use stiffer fabrics, the artist concocted a combination of plain weave layers: textured linen, interfaced poly-cotton, and carton-like beading stiffener. This was used to create a fully functional speaker coil mock up with decorative beading and white cotton zigzag over the conductive thread spiral for optimal UV reactivity. The mock up included all yoke layers and a plastic backing, to cover the speaker's conductive snap connector leads, was applied to the facing fabric with Velcro. Since the 
electronic audio system development was taking place in Montreal at that time, she sent the mock up by courier for testing. Both the consultant and technician concluded that the beading pattern deflected sound vibrations in all directions, which resulted in extremely poor sound quality. Based on their advice, the artist decided against proceeding with beaded speaker coils. Further testing showed that even without the beading, difference in the fabric's surface texture changed the sound. For example, a thinner, poly-cotton plain weave with little surface texture sounded more tinny and metallic while a polyester drill with a slight surface texture sounded rich and organic. While the carton quality of the beading stiffener was beneficial, its thickness acted like an absorbent felt padding, muffling sound vibrations. Eventually she reverted to the same fabric-interfacing combination as had been used in the conductive thread test coil that had initially chosen for its optimal volume and look. She was baffled to find that the newer samples looked identical to the original version yet they did not perform as well. The final version employed the same interfaced polyester drill with a layer of poly-cotton plain weave over for colour matching. The speakers were sewn as appliqués with fake fur trim to create a dandelion clock effect and the interior was prepared with conductive snaps and plastic panels.

\subsection{Working with Consultants: Evolution of the Wearable Audio Electronic System}

Several consultants contributed to the development of the wearable audio electronic system that would be used in the final installation. The main components requiring external consultation were an electronic audio amplification, digital recordings, and a handheld magnetic cone. At times, consultants worked solo while at other times they worked with the artist or with one another. Each "expert" consulted held a unique perspective, but perhaps lacked expertise in other intersecting domains, which contributed to difficulty in achieving mutual understanding along the way. Long distances between Toronto, Montreal and Hamilton also posed enormous 
challenges for working with these consultants. Therefore, communication was a crucial factor in the progression and success of each component. The modes of communication employed in each interaction differently affected the artist's experience and outcomes when working with these consultants.

\subsection{Electronic Audio Amplification}

An electronic media specialist in Montreal was hired to improve the volume of the wearable speaker system. First the consultant, who had an impressive list of past sound installations, met with the artist in person to look over the test audio systems. Before leaving her with a number of sample coils and system components to test, the artist discussed the mandate for the project's functionality, timeline, and expectations with the consultant. Email and social networking messenger service were used as principal modes of communication to discuss many concerns and findings during the project's evolution. Often after a mutual discussion, the consultant worked on her own building an amplification prototype. The speaker coil modifications were expedited by courier for the consultant to test with the new system. Online youtube.com video clips, SMS, or via telephone calls were used to elucidate important audio results. Progress was often delayed until both parties could be present because of misaligned interpretations that required extra verification. For example, each person obtained different multimeter readings and physical measurements for the same coil sample so it became crucial to observe and agree upon methods to rule out other variables. When using identical methods it became obvious that with wear over time loosening threads on the sample coils resulted in louder volumes.

As the working prototype of the amplification system for a single speaker coil expanded to be used with multiple units, further research and development incurred more delays. Timing of 
the development was, in fact, very difficult to anticipate. A 10-hour work estimate by the consultant quickly multiplied into 30 hours over 4 months. The consultant's initial order of Printed Circuit Boards (PCBs) had to be exchanged for smaller versions because it was impossible to "hide" these oversized (approximately 30 centimeters in diameter) electronics under the yoke. Smaller PCBs, originally purposed for peripheral desktop computer speakers, were ordered but were similarly too large, stiff, and unsophisticated to be truly "wearable." Each time a new PCB model was selected, complications arose and new perimeters needed to be imposed for optimal resistance (Ohms). This meant that speaker coils needed to be recalculated for size, sewn in new fabric, and tested for accuracy. To circumvent an impasse in her work while the consultant refined the audio amplification system, the artist chose to modify the Jingle Dress design. With the speakers now in a separate yoke accessory, technical confirmation for the amplifier PCBs would be possible at a later date and transportation for testing would be more convenient. The consultant was able to customise four mono channel PCBs that were equipped with two audio outputs per board, a total of eight mono audio jacks. The four boards were connected in series to a single power jack. Each speaker was equipped with two hand sewn nickel snaps, one positive and one negative. When connected with the nickel snaps soldered at the ends of cables, these relayed the mp3 signal through the jacks to the speaker coils. Separate mp3 players with built-in amplifiers also connected to an audio line-in jack on each PCB. The mp3 players seemed ideal because they were relatively inexpensive, easy to program, and were specified for low voltage draw. Despite compelling progress with the amplification system, the deadline for the installation was quickly approaching with more hurdles cropping up.

The electronic media consultant restructured the PCBs to better satisfy specifications. Due to time constraints and level of voltage required, it was agreed that the audio system would 
be powered by voltage from a wall jack. This impacted mobility for a wearer because the power cord would literally anchor them within its limited range. However, in a museum setting where staff would otherwise be required to monitor and recharge batteries, this would be a logical preference for a more basic power functional. Next, she reduced the physical thickness of the PCBs by crushing the aluminum heat sink and folding the leads on other soldered components so they would lay flat. This achieved an approximate PCB thickness of 2 centimetres, width of 6.5 centimetres, and length of 10 centimetres (Figure 8).

The modifications successfully reduced the dimensions of the PCB, but also produced several unwanted results. Primarily, it caused short circuiting and soldering breakage to a PCB, which resulted in three irreparably damaged mono jacks. Secondly, crushing the heat sinks caused the units to generate extreme heat by preventing proper air venting and heat dissipation. This posed both a safety risk and a fire hazard to humans and museums alike. The artist changed one heat sink for a more efficient version, but it did not yield sufficient cooling to function safely as a wearable electronic device. At this juncture, the consultant recommended constructing plastic containers to house the PBC units. Since endangering the garment and further delay were both out of the question, the artist opted for longer cords (183 centimetres) that would allow the PCBs to simply be placed in a secure location on the floor away from the garment instead.

\subsection{Digital Recordings}

Another consultant, a sound technician, aided in the recording and mastering of digital audio tracks in the last two weeks leading up to the installation. Prior to the actual recording session, the technician had met with the electronic media consultant to familiarise himself with the audio system and fabric speaker coil prototypes. His professional insight was timely in that he not only helped the consultant trouble shoot minor technical difficulties with the audio gear, 
but he also made important material recommendations for the speaker coils. The electronic media consultant had overlooked that a particular range of frequency was necessary for the speaker coils to work at optimal volume. Having different experience and perspectives on sound, the technician used an oscillator to establish that high frequency electric signals (over $100 \mathrm{~Hz}$ ) demonstrated a higher decibel range, and more audible volume level, than low frequencies. Other important suggestions he made were the need to amplify the speaker's potential for increasing vibration and to focus the directionality of its vibratory resonance. This was achieved by creating an air space in the yoke between the rear surface of the coils and a hard, seal-coated plastic backing (Figure 7).

The artist and technician both contributed the 'live' recording session. In preparation, they examined different combinations of spaces, microphones, and manipulations of the Jingle Dress to compose an ideal set-up for recording. Finally, in a small, closed room with curtains to dampen echoes, the technician used an iPad to record while the artist danced and shook in her Jingle Dress. While the Jingle Dress Dances typically only have two or three different drum beats to regulate a dancer's steps, a larger variety of movements were used for these recordings to create interesting sound pattern samples. The technician also recorded a dictation of the artist's poem "The Noisiest Dress in the Quietest Place.” These samples were later composed into remixes and mastered into tracks for the mp3 players to play back on the speaker coils.

Communication for these compositions was mainly carried out via email, social networking message services, or telephone. Nonetheless, their process of reviewing digital sound files had a similar advantage to that of working in-person. By telecommuting in real-time, immediate decisions concerning stylistic and technical modifications could be made. The swift momentum of their ongoing conversation stimulated creativity, enthusiasm, and a continuously 
productive workflow. Errors and misunderstandings still arose on occasion but were dealt with promptly. For example, when technical vocabulary caused confusion, they used digital time stamp references to describe sound preferences, which granted a more precise understanding than if they used layman's terms. Due to the fact that multiple versions sounded similar, they had to strictly adhere to a mutually defined file naming system to avoid confusion. Finally, in testing the final tracks the artist noticed something wrong; they had been mistakenly mastered in stereo. Luckily, the sound technician was there to provide the necessary support to rework the masters for mono jacks, as previously determined.

\subsection{Handheld Magnetic Cone}

Although the magnet was not the principal focus of the installation, it was equally important in the interaction required for the wearable electronic device to produce sound. In early stages of testing, the artist held up a large, 2.5 centimeters rare earth magnet to the speaker coil with her hand. Later on, five magnets with 1.3 centimeter diameters were stacked together to magnify the overall magnet field strength for greater electromagnetic inductance. Both the electronic media consultant and the sound technician recommended that the magnets could be attached to a cup shape. They explained that it would increase the amplitude of sound vibrations produced much in the same way as a child's homemade plastic cup and string "telephone." Finding, or designing, a cup-like container for the magnets that was relevant to the interaction was another task in and of itself.

The handheld magnetic vessel underwent several aesthetic and functional evolutions before its final iteration. Prior to the technical recommendation to use a metal cup shape, the artist had considered embedding the magnet into a "talking stick." The talking stick is a ritualistic item developed by aboriginal peoples to facilitate group discussion in a democratic 
manner. When a person accepts to hold a talking stick, they assume authority to talk, and then pass it in turn to the next speaker when they have concluded. The idea of a visitor assisting the artefact to tell its story via a talking stick interaction was compelling at the time. However, material challenges and the technical integration of the cup shape created a conceptual paradigm shift.

The artist designed several iterations befitting of the materials she contemplated working with. Bone would allow the magnets embedded within it to vibrate while the surface could be carved with decorative elements of the dandelion. However, not many local sources could provide a bone with dimensions adequate enough to create a large cup diameter. Then she consulted with a carver in Northern Canada who suggested adapting the idea for soap stone, which in retrospect did not match with her original vision. Since a magnet will naturally adhere to steel, and placing it on a small steel plate will dramatically increase its magnetic force, a steel cup with a flat base for magnet placement seemed suitable.

The next step involved seeking help from a local metal sculptor to render a steel vessel. Initially, the steel cup design was narrower at the base and gradually curved upward into a wider horn shape, much like a gramophone. Held in the hand next to the garment's speaker coil, vibrations and sound from the magnetic end would, in theory, be emitted louder from the cup shape directly facing the visitor. Working together in the metal sculptor's studio, the sculptor welded steel strips that had been cut according to the artist's aluminum foil design mock-up. Since the final product was tube-shaped, the sculptor welded a small plate inside the narrow opening as a base for the magnets. Upon experimentation with the electronic audio system and speaker coils, the stainless steel vessel yielded poor results. Not only was it not particularly reminiscent of the Jingle Dress aesthetically, but its size and weight were cumbersome. 
Furthermore, the combination of the metal's thickness and the positioning of the magnets in the open end impeded vibrations (sound) from being transmitted effectively.

After much deliberation, the steel vessel was abandoned for a single Jingle Cone, stuffed with a cache of magnets and capped with a thin steel plate for additional magnetic strength (Figure 9). This was an effective tool for uniting the visitor and garment because of its simplicity and ease to manipulate. As a smaller part of the whole artefact, the single Jingle Cone element would not distract attention away from the valuable experience of the artefact. Rather, it represented a complementary touch-object not necessarily to be perceived as a replica. Furthermore, the thin tin metal was effective for amplifying sound. However, since the wide end of the cone focused vibrations back toward the garment it became more obvious to visitors that sound was actually coming from the fabric speaker coils.

\subsection{Installation}

The installation Seeing Fashion through Sound took place on April 2, 2014. Throughout the process of its conception and execution, three overarching themes rose to the forefront: environment, audience, and interaction. Namely, it was the instigation of a sensory experience through the staging of a physical location with particularly stimulating ambient elements. Simultaneously, visitors from many diverse communities were invited to interact with the installation. To receive this audience, a number of accessibility inclusive provisions were thus made to accommodate visitors. During the installation, the artist noted observations of visitor relations with the artefact. These exchanges were, by far, the most potent in revealing insightful situational aspects. Moreover, it was especially the "unexpected" visitor-artefact interactions that now serve as meaningful evidence on which to base future recommendations. 


\subsubsection{Staging the Environment}

In staging the installation at Ryerson University, careful attention was allotted to three main attributes: choosing a physical location, coordinating with institutional administration, and designing a thematic, multi-sensory set. The installation was located on the basement level of Kerr Hall West inside an enclosed stairwell lobby, a traffic junction where many students and staff crossed regularly. This site was the most ideal, available, and accessible for hosting the vast range of physical mobility since visitors could approach via an adjacent stairwell, elevator, or wheelchair accessible ramp. Signage was placed at each access point to indicate access routes. Additionally, a large poster and an information table were exhibited in the lobby to advertise the installation taking place. Because visual signage is not adequate enough to guide people with low vision, one blind visitor requested specific directions in advance by email. Once at the central lobby area, a visitor would be ushered through a second door to experience the installation. the area behind this door, which under normal circumstances was a wide, brick-walled, antechamber leading to an office and a photo studio beyond, was adapted into a temporary isolation chamber.

Advance reservation of this space entailed a number of approvals, precautions, and compromises as outlined by Ryerson University administrative personnel. In total, four different levels of institutional infrastructure were consulted: the administrator for the Fashion Design Program, Fashion Department support technicians, the director of the Fashion Graduate Program, and Ryerson University Security. The Fashion Department support technicians provided a single option; the installation was to be hosted inside a small room located within a graduate classroom on the second floor of the same building. From the artist's perspective, this location was unsuitable for a number of reasons; primarily it was more difficult for low-vision visitors to find and for low-mobility visitors to access. Furthermore, the influx of visitors to the event would 
interfere with classes held in the room. Vice versa, noise from the classroom would filter in, disturbing the installation's soundscape. Finally, the Fashion Graduate Program Director recommended the enclosed passage location, which seemed extremely suitable in terms of creating the dark, quiet space needed for the installation.

The Fashion Department support technicians were initially resistant to the idea, citing the possible conflict with fire regulations. After much negotiation between the Fashion Graduate Program Director and the support technicians, it was concluded that the installation could only take place at this site under certain conditions. These conditions stipulated that the setup, installation, and removal of material could not surpass a four hour period and was to take place after class time. Furthermore, building security had to be notified in advance in case of an emergency and the passage was to remain locked when not in use. The artist had to once again rely on the support of the Fashion Graduate Program Director to vouch for her with the Fashion Design Program Administrator in order to obtain provisional permission for a key to the passage. At this time, Ryerson University Security was also informed of the temporary situation in the event that a contingency plan for evacuation would be necessary.

Several depth-enhancing elements were then arranged inside the window-less antechamber to create an ambient background. The exhibition space was separated by a black curtain to filter out external light and noise from the rooms beyond. To combat the additional effect of airflow that caused the curtain to billow outward, it was attached in rear to a supportive frame. Placing the mannequin further from the entrance acted as an invitation to enter deeper into the immersive environment where the opening of the door would not cause light pollution. In practice, this greatly accentuated the installation's black light illuminated components and attracted visitors inward to interact with the Jingle Dress's wearable speaker yoke. However, the 
depth of the room and placement of lighting and sound components were not the only factors pertaining to the effectiveness of the installation's soundscape.

There was also a great deal of noise interference in the soundscape. In theory, the area should have been quiet with little external noise pollution. Normally, neither the adjacent office nor the photo studio is in use during the early evening. Typically, the lobby is also rarely busy with student traffic at this hour. However, due to the special occurrence of Mass Exodus students photographing their creations regularly interrupted the installation by playing music and talking loudly. Another important environmental condition that affected sound was the echo caused by the small room size and texture material surfaces in the space. Within the narrow passageway, the directionality of the yoke's speakers caused sound to deflect crisply off the hard, smooth brick walls and the metal door at the entrance. Moreover, depending on their footwear visitors' footsteps contributed a variety of additional echoes to the soundscape ambience. These sources of noise pollution resulted in an overall dampening effect of the wearable speakers' sound levels.

In fact, the wearable sound system demonstrated a number of technical limitations that prevented it from compensating for these unanticipated acoustics. Its Mp3 player manufacturer settings capped sound output to levels that would be non-hazardous for earbud listeners; the amplification required for larger speakers and spaces was not possible. Furthermore, the interfacing material and conductive thread both insulated against the formation of sound vibrations. The use of an uncoated plastic backing diminished the speaker's ability to resonate. Sealing the plastic backing with a coat of spray paint could have improved its non-absorptive aspect, hence resulting in better outward sound projection. The Jingle Cone used to electromagnetically induce sound vibrations performed its task well, but could be improved by 
using a different shape. Magnification of the sound would be increased if the shape of this object were cup-like, with the opening facing toward a person, and if it were to be made in thin steel. In this way, a magnet "stuck" to the outside, bottom end could vibrate more readily against the metal cup as it neared the conductive coil speakers. In moving forward, an important step would be to use an environment that is more suitable for the installation's sound system, better isolate it from external sensory disturbances, and to use more appropriate amplification equipment.

\subsubsection{Connecting with the Audience}

Considering the diverse channels used and volume of potential visitors invited to the installation, attendance was surprisingly low. The artist's strategy for promoting the event relied heavily on advertisement via a social media and organisations. Sharing info on a Facebook event page, posting in various special interest groups, as well as emailing several organisations so that invitations would be issued to their members was not enough to incite a larger turnout. Very few from the School of Fashion attended, likely due to the installation's limited timeframe. Some who could not attend cited schedule conflicts from rush hour traffic and participation in Mass Exodus's fashion show. In fact, of the approximately 30 visitors who attended, most were acquaintances whom the artist had invited personally by word of mouth and personalised emails. One exception was that almost every board member from RASS attended. Another group that came out in observable numbers were students of the Creative Industries Program. Through their interactions, comments, and questions members of these groups demonstrated more interest in the cultural work and sensitivity toward its representation than people with a purely fashionoriented interest. 


\subsubsection{Observable Interactions}

The artist greeted each visitor in the lobby to make them feel welcome and to prepared them for their voluntary participation in the installation. Individuals could learn about, touch and manipulate a mock-up example of the wearable speaker technology at an information table. In fact, this was intended to inform visitors about the technology used, and to subtly train them to produce optimal sound results through their interaction with the speaker coils. The beaded speaker sample was used to emphasise how an irregular surface texture could affect sound emissions. The artist demonstrated that the clearest and loudest sounds resulted, not from touching the magnetic cone to the coil, but by keeping it approximately five millimetres from the coil's surface. Many visitors concentrated on performing interactions with the coil, whereas only an exceptional few preferred to inspect the coil's aesthetic qualities instead. The latter commonly expressed a perception of difficulty related to its embellishment beading. Visitors were notably less concerned with technical or functional aspects of the wearable speaker mock-up.

Next, small groups of visitors entered the antechamber and began to interact with the installation, one person at a time. The artist accompanied each group to observe how each person engaged the artefact in the immersive environment. Despite the preceding "no touch speaker training," visitors often made direct contact between the cone and coils. Even so, direct contact between the two still projected enough audible sound for visitors to listen to different recordings of the Jingle Dress sounds and the poetic, spoken reflection on accessibility in the museum of fashion:

The Noisiest Dress in the Quietest Place

I beg you tell me your story.

My eyes are closed and I listen, 
Yet there is no sound.

I hold a tool.

Yet there is no sound.

I have the power,

Yet there is no sound.

The noisiest dress in the quietest place.

I beg you tell me your healing story.

My eyes are closed and I listen,

Yet there is no sound.

I hold a tool.

Yet there is no sound.

I have the power,

Yet there is no sound.

The noisiest dress in the quietest place.

Additionally, occasional background noises from the adjacent studio drowned-out the sound from the installation. To compensate for this, one visitor almost instinctively placed the cone's tip to her ear to isolate the coils vibrations from the competing noise. In doing so, she essentially achieved additional volume amplification. Another unexpected interaction occurred when a fashion student took the initiative to lift up the skirt of Jingle Dress to inspect the reverse. She claimed that she wanted to see how the garment finishing was done inside.

One visitor who was a blind, and another who arrived in a wheelchair, engaged in remarkable interactions with the artefact. At first, the blind visitor was unable to localise the center of the speaker coils before employing his sense of touch. He initially attempted to locate 
the site as its position was described to him, waving the magnetic cone erratically around the mannequin. Other Jingle Cones on the dress were attracted to the magnetic cone, so pulling it away from the dress required forceful detanglement. With the use of his hands, he was finally able to feel the dress to navigate toward the center of the speaker coils. Thus he was able to position the magnetic cone exactly where it would be most effective and then listened to each. The visitor in the wheelchair was unable to access the point of interaction because the mannequin's chest height placed the speaker coils out of her reach. In reaction to the installation, visitors typically had questions and comments leading to a discussion about visual accessibility issues. With this last visitor, the artist shared an anecdote about searching for a seated mannequin for the installation. In a whole warehouse full of storefront mannequins, she had been unable to find any seated mannequins. However, the visitor in a wheelchair, who also worked to provide accessibility for Ryerson University students, deemed that the provision of a [touch] screen at her seated height would have been a sufficient solution. 


\section{CHAPTER 5: RECOMMENDATIONS AND CONCLUSION}

In this concluding chapter, the research of this study will be summarised for the purpose of offering a number of recommendations, for the consideration of Ontario fashion and textile museums as they respond the AODA mandate. Firstly, the contextual limitations of this study will be reviewed. Secondly, the initial research questions, which were based on a conceptual extension of the AODA mandate, will be revisited alongside the major findings of the study's creative component. Through this retrospective, important contributory factors and relevant outcomes of the study's interactive installation will be carefully weighed and interpreted. Thus, the set of recommendations, based on the artist's unique Métis perspective, will be formulated to contend with the current deficit of inclusion and accessibility in the museum of fashion. The intentions of artists, institutions, and the public they serve will each be evaluated in accordance with their potential for participation in the synthesis of new exhibition protocols. Particular attention will be made in reference to new opportunities for creating complementary sensory and cultural experiences with clothing artefacts. This chapter will also address recent examples where the interpretation and execution of accessibility initiatives has resulted in newsworthy conflicts. This will outline some of the current challenges faced by cultural institutions in their aim to fulfill the legal requirements of the AODA mandate and in satisfying public interest within the next decade. Ultimately, the conclusions of this study will address annexes for future academic research in the spheres of inclusion and accessibility for the museum of fashion.

\subsection{Limitations of the Study}

In light of the 2025 AODA mandate, the objective of this study was to explore the museum of fashion's limitations in its ability to provide accessibility and inclusion. While the AODA legally requires public institutions to revise to built-environments and to restructure 
services to accommodate people with disabilities, this study was largely targeted toward creating new and authentic interactions with a clothing artefact. The interactions and artefact, situated within an artistic installation, resulted in stimulating experiences. With the installation of several multimedia elements, such as the modification of the visual environment and soundscape, the artefact was further contextualised in such a way as to intensify the visitors' perception and experience. The study itself was limited to the artist's experience of the creative process she chose to employ in the creation of a digital media installation and a number of interpretations she derived from observing visitors interactions over several hours. The installation took place in April 2014, at Ryerson University in a temporarily reserved hallway. There, thirty-or-so visitors were observed as they interacted with the sound-emitting digital electronic components of her clothing artefact.

For more accurate study results, it would be ideal to exhibit this interactive installation for a period of several months in an Ontario fashion museum. The exhibition of important clothing artefacts in museums such as the Royal Ontario Museum and the Textiles Museum of Canada located in Toronto, and the Fashion History Museum in Cambridge, is supported by numerous internal and external frameworks. For example, these public institutions are eligible to receive government subsidies to invest in exhibition technology to accommodate visitors with disabilities. More funding is available for developing exhibition technology and hiring media consultants, which results in the production of a more compelling immersive and interactive visual and acoustic environments. Access to similar funding and technical support from consultants would allow for the implementation of faster and more complex technological developments such as miniaturisation of components, alternate sound amplification technologies, and thus, more effective interactions. High profiles in social media and other well established 
channels for the diffusion of advertisement and promotional marketing attracts a higher visitor count from a wider range of demographics. This reveals a more accurate snapshot for the inclusion of disability and indigenous populations than was possible through the reach of artist's own social and professional networks. Furthermore, studying the artefact's electronic and material components over several months of exposure to manipulation for visitor interaction, professional research, and ritual use could lead to other important recommendations. A lengthier exhibition of the installation in a fashion museum setting would ultimately provide a loftier bank of observations pertaining to visitor experiences. Not only can more salient conclusions can be drawn from this, but it could also provide relevant data to drive the creation of new curation protocols for emerging interactive media exhibition technologies.

\subsection{Summary of Study Results}

The artist was able to draw a number of conclusions regarding the study's initial research questions from her personal experience of the creative process and installation. Prior to engaging in the study, she defined different types of planned interactions that occur in the museum of fashion, and more precisely, interactions that are discouraged or exclusionary. For interactions that are encouraged, she also considered the value of a visitor's experience with authentic clothing versus replicas. In cross-referencing these findings with the gap in the fashion museum's ability to provide equal opportunities for visitors to interact with authentic clothing artefacts, she was able to identify a population that is currently being underserved by this type of public institution. The AODA mandate also recognises this important statistical increase in disability demographics, and thus, it identifies major areas for inclusive adaptations in public institutions and legally requires them to meet a number of accessibility standards. In considering the initiatives outlined by the AODA mandate, the artist began to consider a more 
comprehensive extension of its concept. This brought her to reflect on how fashion museums will plan for further inclusion and accessibility that will encompass visitor-artefact interactions and experiences. The first research question: "Which visitor special needs could be better accommodated for in the museum of fashion?" helped guide the study to identify who the target of this study might be.

In fact, there are vast numbers of people who could benefit from upgrades to the framework for inclusion and accessibility in the museum of fashion. Since these institutions of material culture chiefly provide means for visitors to view artefacts inside a protective, lowly-lit, “do-not-touch" display case, this systematically excludes visitors with any number of visual disabilities. For low-vision, any visual factors in the environment that are less-than-ideal, such as improper distance, lighting, contrast, skewed perspective could be disadvantageous to the viewer's perception. In the case of complete blindness, no visual environmental can be ideal and prevention of touch interactions with an artefact can be disruptive to a visitor's experience. In yet other circumstances, a person's eyes may be standard but the operation of sensory relays between their ocular structures and brain can result in atypical interpretations of sensory information. In all of these cases, adding complementary types of sensory interaction with artefacts could make a world of difference in terms of visitor accessibility to experience. Hence, policies for improvements to the museum built-environment and visitor services are important, but investigating potential accommodations to meet the sensory needs visitors with visual disabilities is also paramount. Furthermore, the urgency to remedy the current situation is compounded when considering Canadian demographic segment growth for people with visual disabilities, especially due to projected increases low-vision in aging populations. 
The artist also identified another instance of problematic protocols in relation to inclusive cultural accessibility. This became a factor during the creative component of the study due to conflicting views about her artefact's cultural value and the representation of a sacred item in this installation. Through discussions with other members of First Nation groups, she began to better understand the specific cultural need of indigenous cultural groups to re-establish ownership rights to ancestral items that are held by museums. Whether the items are deemed to be sacred or not, there is a push from indigenous groups to decolonise museum policies, which would allow them to access their heritage items for ritual use and preservation. Many indigenous groups are committed to the development of new museum protocols that would allow designated group representatives to participate in decisions pertaining to the appropriate care and representation of their cultural heritage artefacts. Although the systematic-exclusion of access to artefacts in museum has prompted indigenous groups to use replicas for the education of future generations, a complete experience with authentic artefacts still requires vast improvements to accessibility protocols.

The second research question of the study: "How can museums of fashion employ digital technology to curate inclusive, accessible experiences with artefacts?" focused on circumventing the museum's no touch policy. Firstly, the use of peripheral digital media support and replicas as a curatorial method was ruled out because the superior aura of an authentic artefact would provide a more powerful interactional experience for visitors. Considering both the visitor's need for edutainment and the museum's protocols for preservation, the artist planned for the use of a no-touch electromagnetic induction "switch." By embedding multiple conductive speaker coils directly into the garment, the artefact itself then became the sole source of sound interaction. 
Thus, visitors could use a magnetised Jingle-cone to initiate a number of digital recordings to be played back through the dress without touching its surface.

However, the installation also focused on generating awareness about accessibility in the museum of fashion by exploring exclusion to experience. The sensory components were not intentionally exclusionary of blind visitors or visitors in wheelchairs, but some elements were developed specifically as a comment on accessibility gaps in exhibitions of visual culture. The intention was to even the playing field, so to speak. For example, black light was employed to effectively reducing visual information from the garment within the immersive environment of the installation. Even when their vision adapted to the darkness of the installation, black light was a disadvantage for most visitors. Essentially, a person with typical visual abilities could feel the perceptual disadvantages. The experiences included a difficulty to discern garment details that were non-UV-A reflective and the difficulty to guess the garment's colour. Visitors often were surprised to find out that the reddish brown and violet fabric was actually yellow and white under full spectrum visible light. However, these same UV-A lighting elements that created a situation of exclusion to visual experiences for people with average vision were also put in place to give an experiential advantage to people with low-vision. High contrast UV-A reactive elements theoretically improved visibility for people with low-vision. Furthermore, a visually exclusive UV-A lighting environment shifted the focus of the environment to the experience of sound.

Another factor of exclusion in the installation was the discreet nature of the interaction. Displayed statically with no informative signage about it, the artefact's embedded electronic functions or mechanical capacity to produce sound would not be accessible to visitors. Nothing in the installation indicated that the coil embellishments were sound producing devices. In fact, 
the customary no-touch exhibition protocol would prevent a respectful visitor from discovering the artefact's full potential without guidance. For this reason, interactive technology "training" was an integral part of inform visitors how to access to the digital sounds. Furthermore, although coils were different sizes, there were no other visual cues to stimulate a visitor's curiosity to explore the variety of different sounds and recordings that could be experienced from each speaker coil. Hence, a visitor's experience was highly dependent upon their depth of personal interest and commitment to spend time examining the installation.

In essence, the curation of an artefact is highly dependent upon how it is contextualised within the museum environment. The last research question: "How can digital technology be used to ensure that a clothing artefact will not be misrepresented through the curatorial process in a fashion museum?" has particular potency for curation of material culture. Museums are extremely vulnerable to accusations of misrepresenting artefacts, simply because curators are not able to display an artefact without imposing some type of context. If a curator were not part of the group of cultural owners associated with the artefact, he or she might not have a full understanding about it. Herein lies the danger of making decisions about portray an artefact in a context that may be largely incongruent with the social-political views, historical records, cultural values, and spiritual beliefs of that group. Decision to use an inappropriate technology and or sharing incomplete or faulty information builds an improper perspective about an artefact.

This Métis artist is not part of the specific group of Ojibwe cultural owners, and the exhibition of a Jingle Dress proved to be problematic for some. She had a personal relationship with the artefact and complete power over how it was presented to visitors. From a technical perspective, lighting and acoustic elements of the installation were quickly and effectively altered using digital media and electronics. The artist selected sounds of her Jingle Dress and 
poem for audio recordings, and then directed the digital mixing. She easily changed the visual representation of her garment with lighting. Finally, visitors were taught how to interact with the installation and shared her personal views of the accessibility with visitors who were interested. Despite, having the best intentions for presenting an artefact in the installation, it could still be identified as a misrepresentation of a sacred Ojibwe item. Key arguments for this were the aberration of using digital technology in the "traditional" Jingle Dress, and the inappropriateness of exploiting a sacred item for exhibition to non-First Nations individuals. It seems that no application of digital media, in and of itself, can solve the underlying need for cultural awareness and sensitivity in presenting artefacts. However, by using digital media to demonstrate sound aspects of the Jingle Dress that could not otherwise be perceived in a static museum display, the artist presented a more complete representation of her own experience of it.

\subsection{Recommendations}

This section of conclusions is reserved for the provision of recommendations for inclusion and accessibility within the context of the museum of fashion. The recommendations are based on the results of this creative research study, which explored the challenges faced by institutions, artists, and the public from the artist's unique Métis perspective. The One Spoon, One Dish Wampum Belt contract provides a basis for her attitude on the importance of these relationships in museum of fashion for the achievement of equitable decolonisation and participation: "They have entered into the great league and contract that they will all be one and have one heart, what belongs to one will belong to all" (Sganawadih, 1888, as cited in Bardeau, 2011, p. 443). The goal of the following recommendations is help encourage pro-active and mutually beneficial participation in the generating of new guidelines and exhibition protocols in the museum of fashion. Some of these recommendations specifically promote the development 
of complementary sensory and cultural experiences with clothing artefacts through the use of digital technology.

\subsubsection{Institutions}

As public institutions, museums of fashion have a large role and responsibility in the process of upgrading inclusion and accessibility within their complex frameworks. Some of the aspects of the adaptation they must develop plans for are: cost, timeline, technology procurement and obsolescence, personnel training, communication, working with consultants, as well as considerations for health and safety. Since the inclusion and accessibility of visitor exhibition experiences are not covered in the AODA mandate yet, there is enormous potential for museums to consider for the future. Disability awareness and cultural sensitivity in the curation of fashion experiences are two areas that were identified by this study for prospective inclusion and accessibility restructuring. These areas are particularly sensitive because they involve groups of people who have lobbied for fair and ethical treatment in lieu of past human rights violations. Restructuring museums of fashion to be fully inclusive and accessible for people with visual disabilities and owners of indigenous cultural heritage will likely be a very gradual one. Even with the best of intentions, making changes to the way in which museums of fashion curate and exhibit clothing artefacts will certainly involve a process of trial and error.

European or Western-based perspectives that govern artefact preservation and interactions in Canadian museums necessitate "decolonisation" revisions for future curatorial practices. Decolonisation, in this case, can be interpreted as a methodology for re-examining the curation of experience in museums in order to be able to understand, respect, and better serve the needs of both indigenous and disability visitor groups. Museums that only cater to the interests of the "majority" need to recognise which groups are being systematically underserved. If they can 
achieve this, then they will also avail themselves to a number of major opportunities for valuable community development. In order to accomplish this, institutions must bring members of indigenous groups and disability groups onboard for participation in the decision making processes. By engaging knowledgeable staff with first-hand experiential implications with cultural artefacts, museums will be better equipped to fulfill their role as caretakers. In this way, they may also continue to expand knowledge about the artefact's intrinsic values and meanings. Decolonisation in museums could restore importance to the indigenous guardianship which treats artefacts as living beings; allowing them to be fed, preserved through use, and eventually buried when the artefact's lifecycle is complete. Loss of the original is inevitable, but for now museum resources should be spent on preserving authentic interactions for cultural owners who demonstrate appreciation, honour and respect for heritage artefacts. Decolonisation that includes the perspective of people with disabilities in museum decision making processes would also make it possible to better understand and incorporate an infinite number of complementary sensory experiences in exhibitions, including: ophthalmoception (sight), audioception (hearing), gustaoception (taste), olfacoception (smell), tactioception (touch), thermoception (temperature), proprioception (kinesthetic sense), equilibrioception (balance), and kinesthesioception (acceleration).

In both cases, with the decolonisation of curation and artefact preservation museum protocols, the value of experience will take precedence over an artefact's declining material value. Digital technology platforms will be very significant in transforming the systematic hoarding of decaying material artefacts into the sharing of cultural knowledge through authentic experiences. Caretakers of this knowledge can use digital audio-visual technology to produce and share documentation about the artefact or about the firsthand experience of its creator that 
will remain after the artefact's lifecycle has been completed. In the same way that fire can be shared from one candle to the next, digital files can be made readily available for a vast public for future study without incurring high costs. Thus, the nature of experience with an artefact becomes more accessible through the process of digitalisation. While certain aspects of its physical form will no longer be available, digitalisation immortalises the original, increasing its value. The Museum of Modern Art (MoMA) in New York City embraced the digital essence of an artefact by purchasing of the Pac-Man game code, not the Pac-Man console patent, because it recognises the value of interaction design and the relationship to the game creators (Antonelli, 2013). Perhaps future museums of fashion will choose to acquire the digital codes of historical garments that work with electronic simulators to permit visitors to see, touch, hear, smell, and perhaps taste aspects of an artefact that no longer exists in material form.

\subsubsection{Artists}

For now, the growing diversity of digital media and electronic platforms used by artists to develop their unique creations far supersedes the museum curator's ability to respond rapidly in kind. For this reason, technical consultation, collaboration, and special insurance is required for museums who wish to exhibit wearable electronic and digital components, such as those embedded in the artist's Jingle Dress. While it is not recommended to invest in any particular digital media or electronic platform due to the rapid obsolescence of technological components and applications, investing in collaboration and consultations with the artists or cultural owners is vital. Museums, artists, and cultural owners should be equally aware that they are planning for installations with open-ended experience outcomes. While external documentation can provide factual data about an artefact, the experience of it is very personal for each visitor and should not be subject to heavy socio-political influence or budgetary and technological limitations. In order 
to achieve this, focus must be brought away from the consumer attitude of the entertainment experience. This will require the development of communication and approval protocols between artists and museums to build a trusting relationship about the representation of digitally enabled electronic works of art. Telecommuting would be less desirable in this situation; in-person dialogues between artists and curators early on would better permit both parties to assess spatial, temporal, sensorial, and human realities of a proposed exhibition environment. Decisions pertaining to the execution of an exhibition would thus not be based solely on one person's opinion or understanding, but on a consensual arrangement about the artefact's representation.

\subsubsection{Visitors}

Visitors have a lesser, but equally important role in the strategy for the future inclusion and accessibility reformations in museums of fashion. In fact, it is through ongoing public support that museums continue to exist and evolve. On the one hand, no one who feels excluded should remain silent. As individuals, visitors with special needs should continue to provide valuable feedback and come forward with request for beneficial changes. The voices of these individuals grow stronger when supported by numbers, and the changes they lobby for occur when those numbers reach critical mass. On the other hand, the attitude and intentions of the public must also demonstrate positive intentionality. This means that visitors should adopt an outlook of responsibility and personal commitment, to the local community, as well as to the sharing of knowledge and cultural heritage. By being more interested and involved with the caretaking and documentation of artefacts, or by participating in museum activities and planning, visitors will be giving back to their community and future generations. In addition to teaming up with museums of fashion, a great deal of patience, respect, and positive support will be required 
as museums revise old curation and visitor experience frameworks to become more inclusively accessible to a wider public.

\subsection{Implementation Challenges and Areas for Future Research}

Both the planning for, and the execution of, AODA adaptive frameworks brings forth a number of challenges for public institutions. Since the inauguration of the AODA legislation in 2005, there are numerous mixed messages regarding compliance incentives and enforcement penalties. On the one hand, late in 2014 the City of Toronto fined the owner of Signs, a restaurant catering to the hearing impaired, because the wheelchair ramp he installed for his customers conflicted with municipal pedestrian traffic obstruction by-laws (Kim, 2014). On the other hand, the Provincial Government of Ontario only officially committed itself to the enforcement of legal penalties for compliance violations until 2014. Only one year later, the Government of Ontario has recently announced that it will be reducing the number of compliance inspections. The confusion caused by this incongruity is likely the reason why $60 \%$ to $65 \%$ of institutions hesitate to engage in AODA restructuration (Monsebraaten, 2015). Perhaps implementation guidelines are not yet clear enough, institutions fear that their investments will be in vain if they risk being fined, or the compliance inspection reductions may discourage institutions from taking the legislations seriously altogether. Many institutions cannot adapt until the many rules and specific protocol they are bound by match legal and ethical standards that also need to be reworked and revised. In the interim, the design of inclusive, accessible experiences could be an area of low-risk and high-value return for institutions.

The concept of eliminating barriers to experience and adding complementary sensory interactions in museums of fashion demonstrates enormous potential for future research. Some of these areas that came to light during this study are: blind interactions in the museum, the creation 
of sound interactions to complement the visual, the curation of wearable electronics, and participatory relationships in museums of fashion. In general, people do not perceive an experience exactly the same way; experience can be influenced by diverse sensory processes or by personal cultural understandings. This will require institutions to develop sensitivity for the needs of its public, yet to also foster interdependent relationships with its artists or cultural owners, and visitors. Priorities need to be set on all administrative and governmental levels to create a situation with clearer guidelines, faster timelines, and more serious consequences for failure to provide opportunities for inclusion, accessibility, equality in the museum of fashion. Environment, tools, skills and expertise are just as essential in building these mutually beneficial relationships as listening, understanding, and clarifying expectations. Above all, sharing knowledge through the curation of inclusive, accessible experiences with material culture is the key to its prosperity and preservation. 


\section{Appendix: Figures}

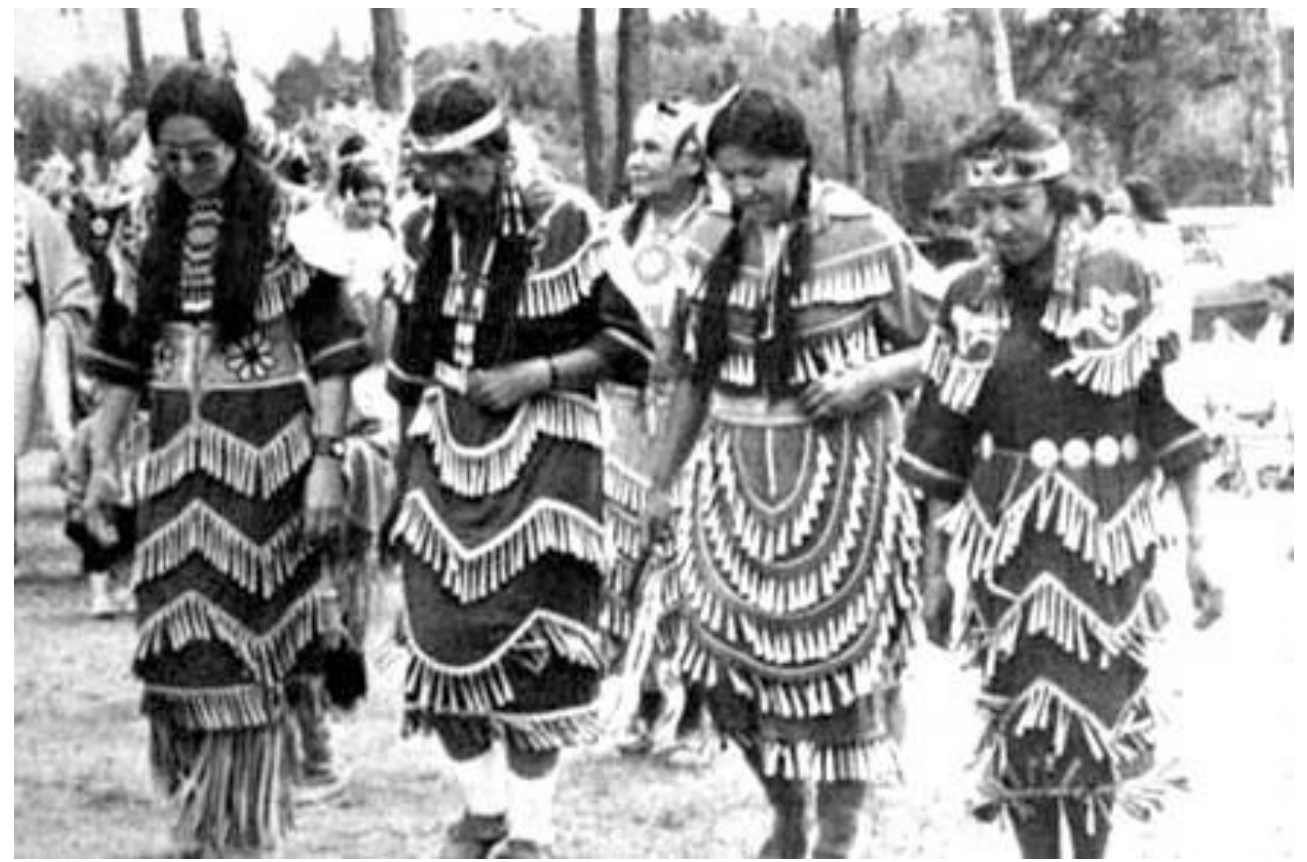

Source: http://www.anishinaabekwe.com/2012/12/sacred-jingle-dress-dance-for-chief.html

Figure 1. Original Red Lake jingle dress regalia style.

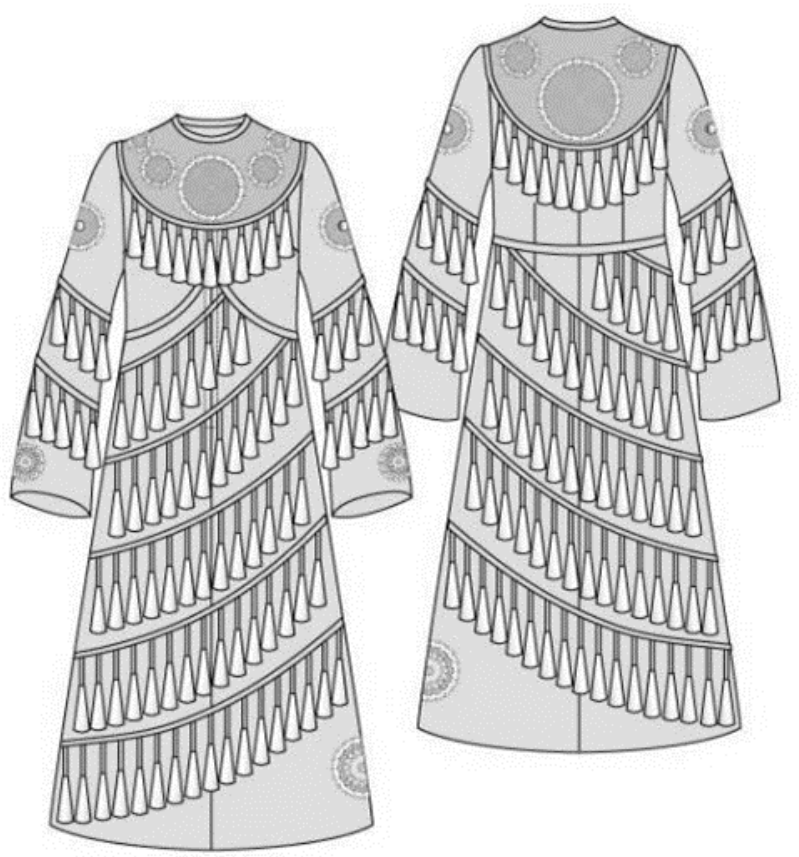

Figure 2. Jingle dress illustration. 


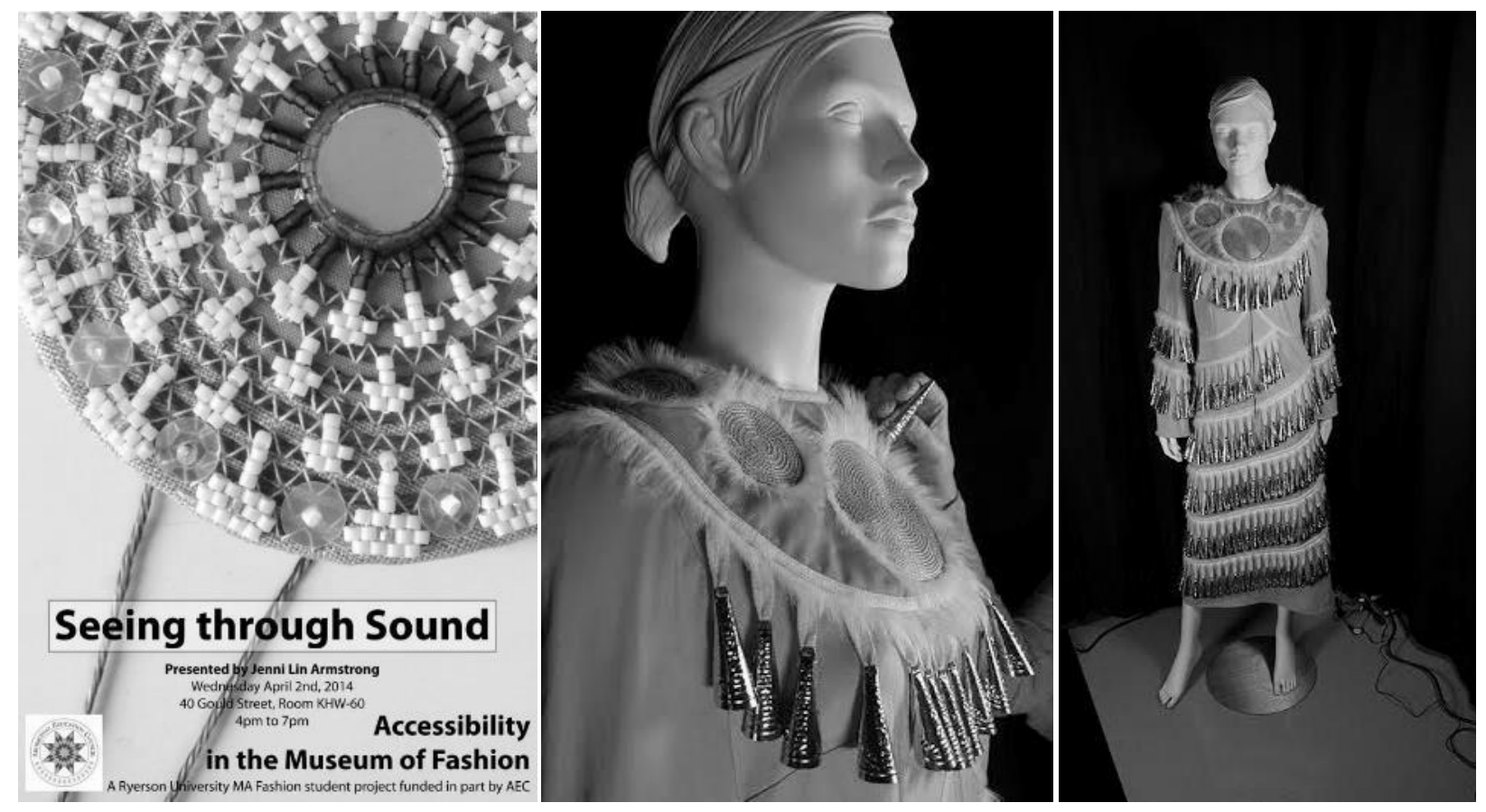

Figure 3. The multimedia installation.
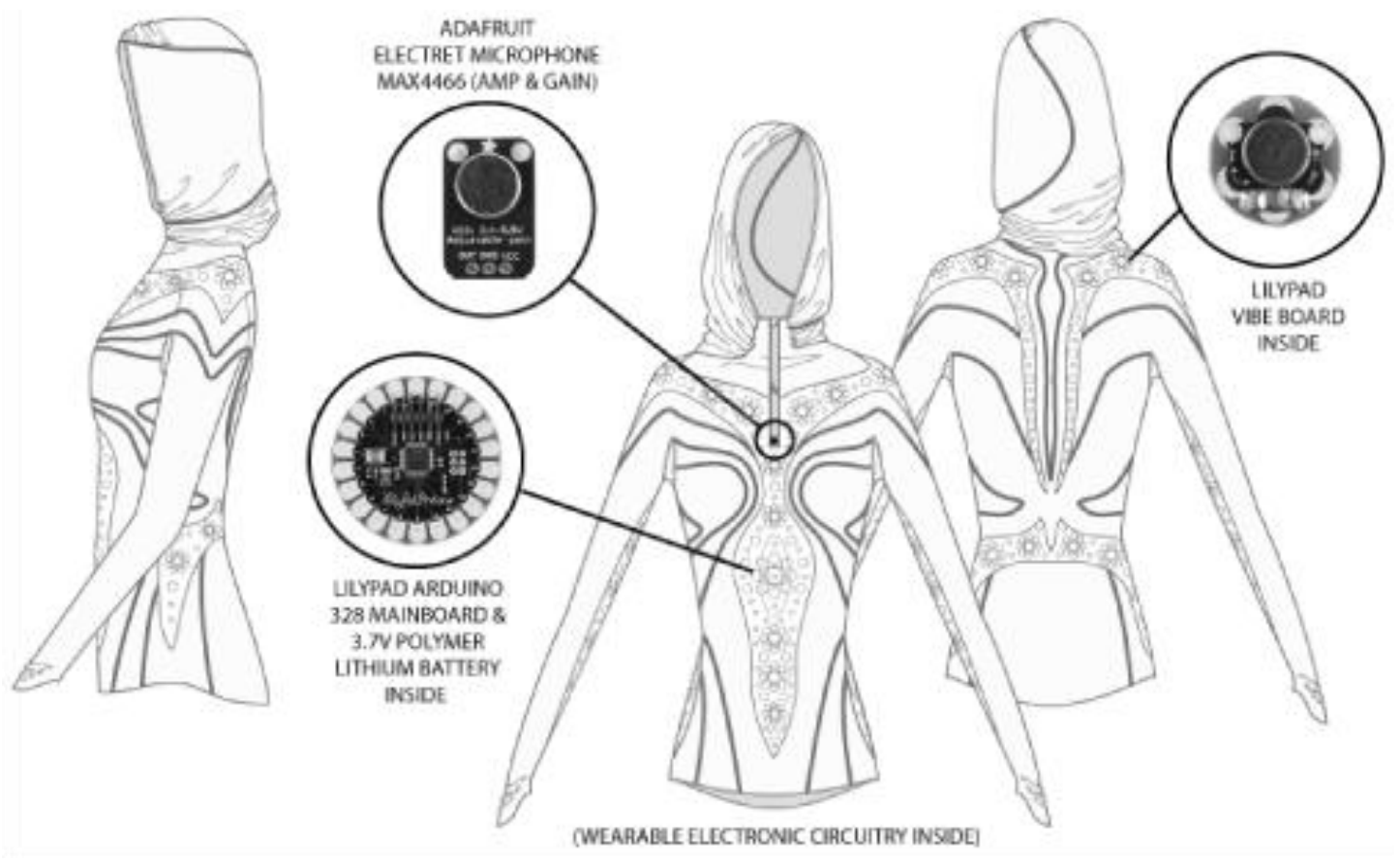

Figure 4. The hearing shirt. 

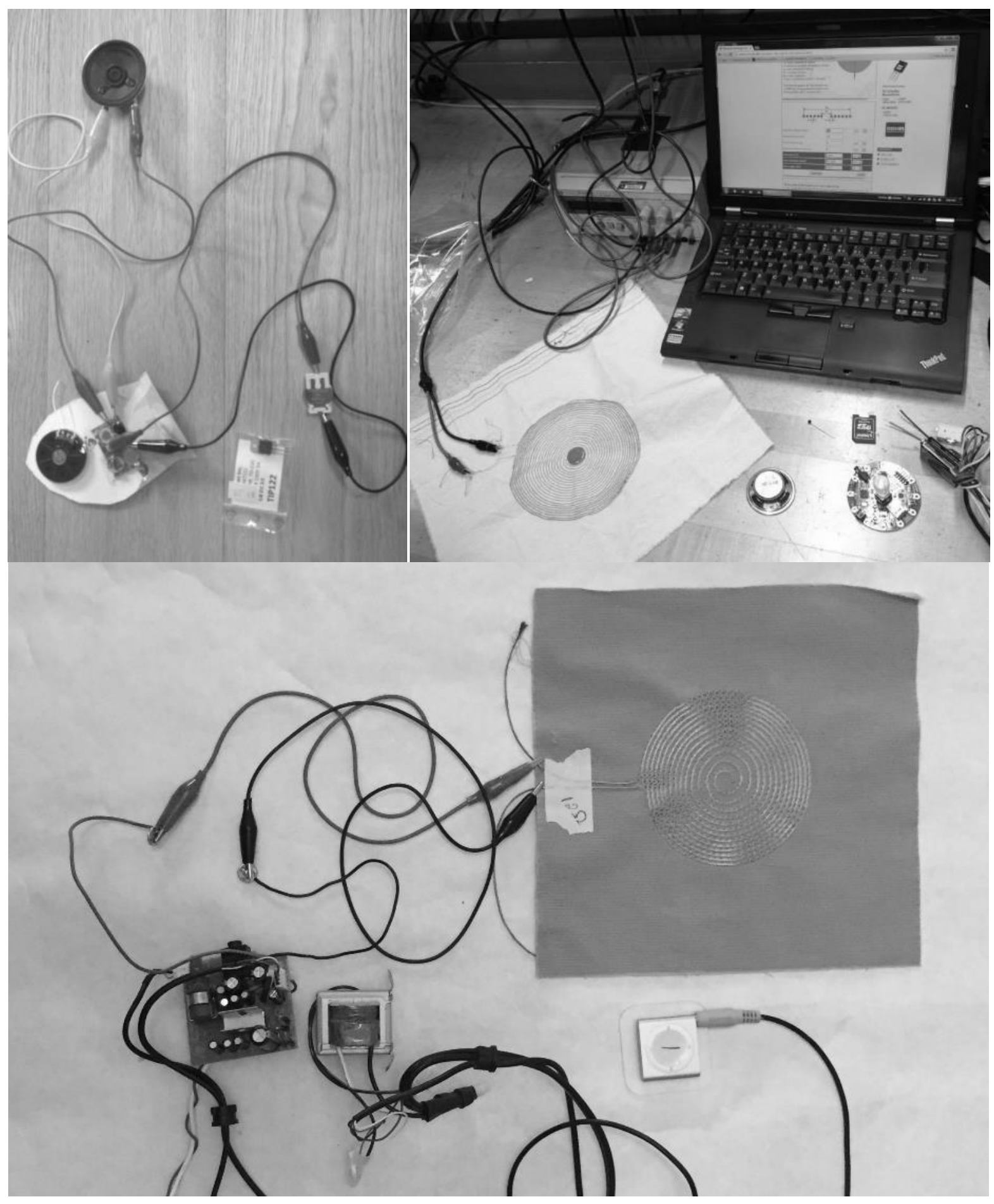

Figure 5. Audio tests. 

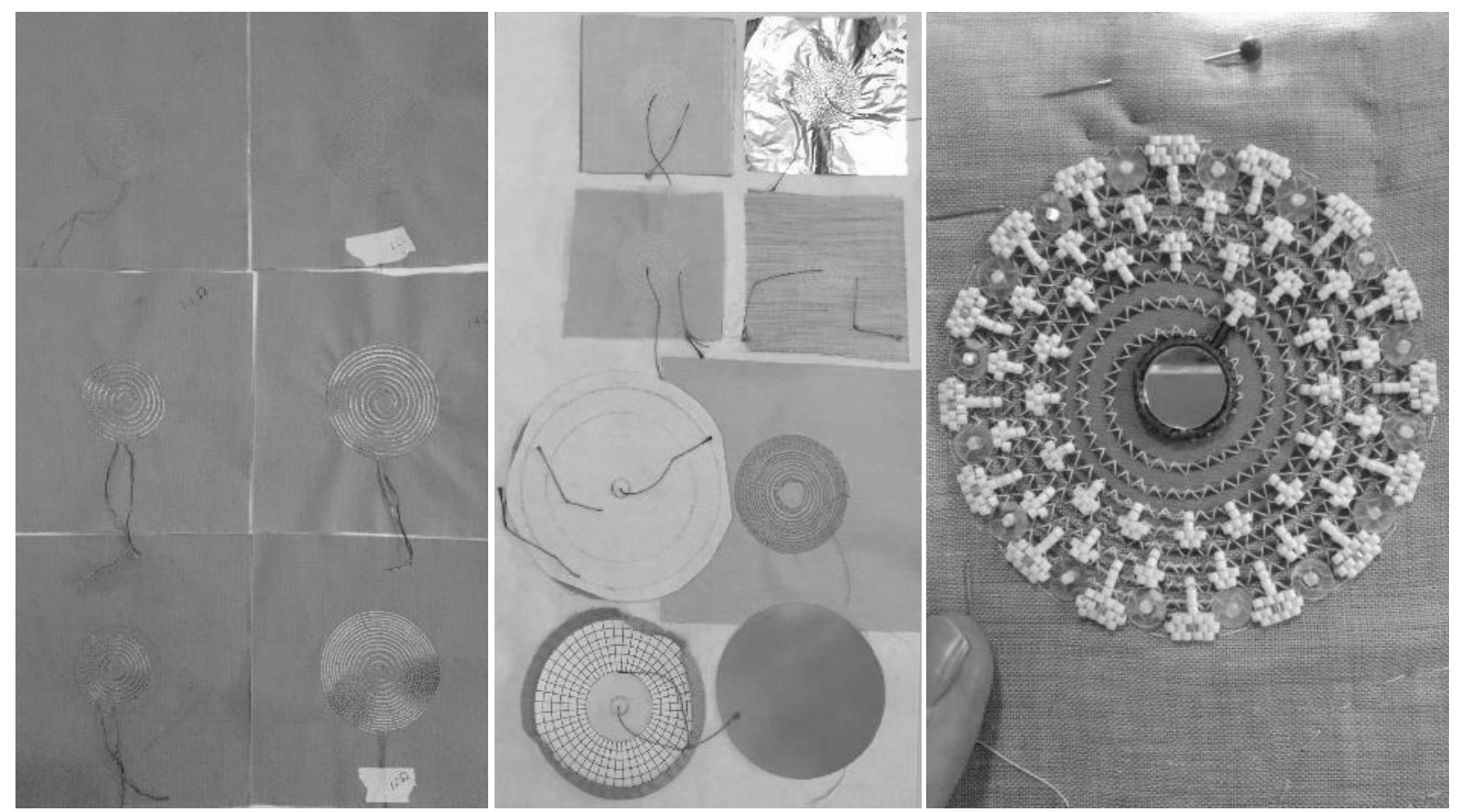

Figure 6. Speaker coil construction and materials considerations tests.
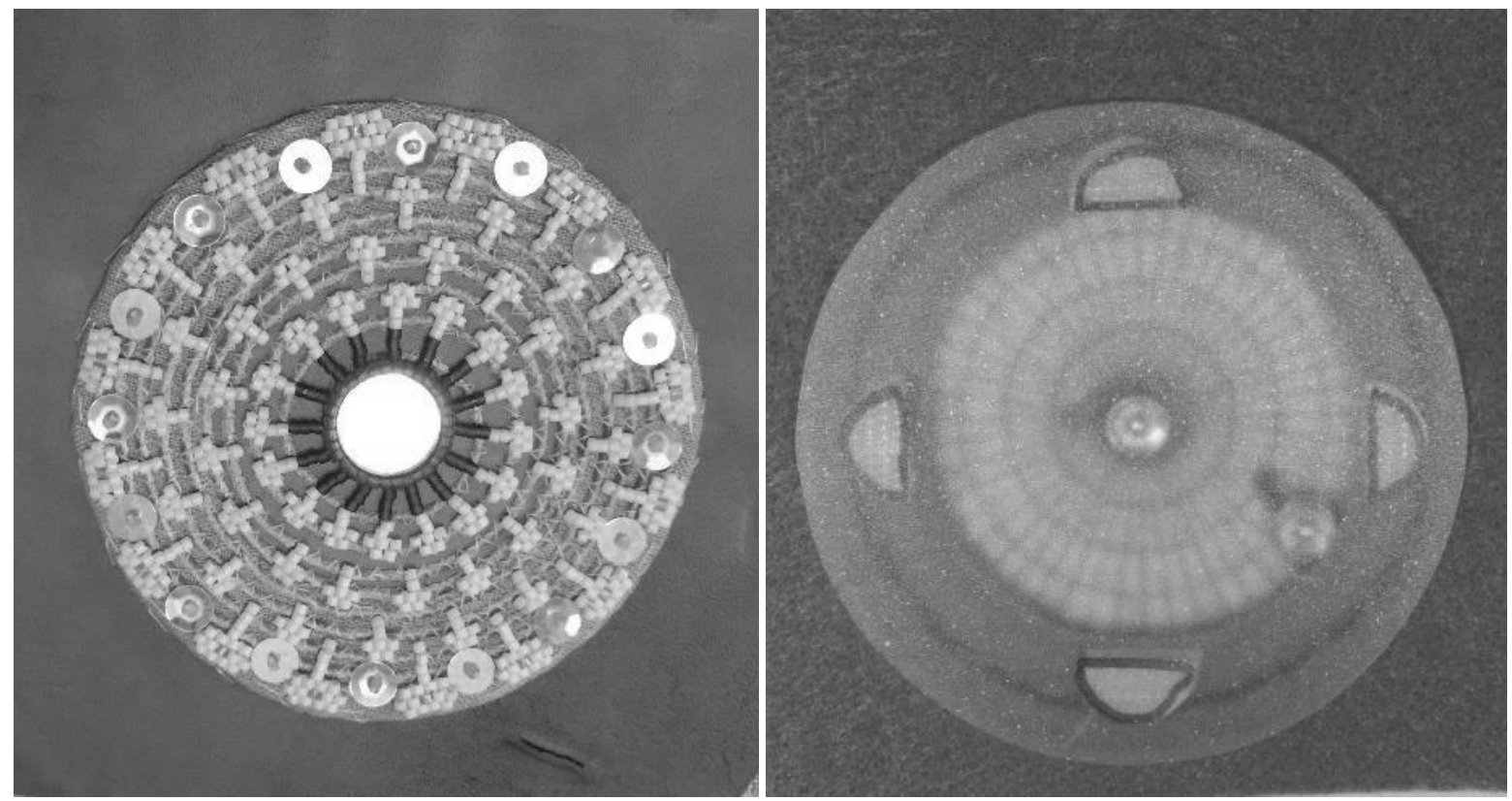

Figure 7. Beaded speaker coil. 


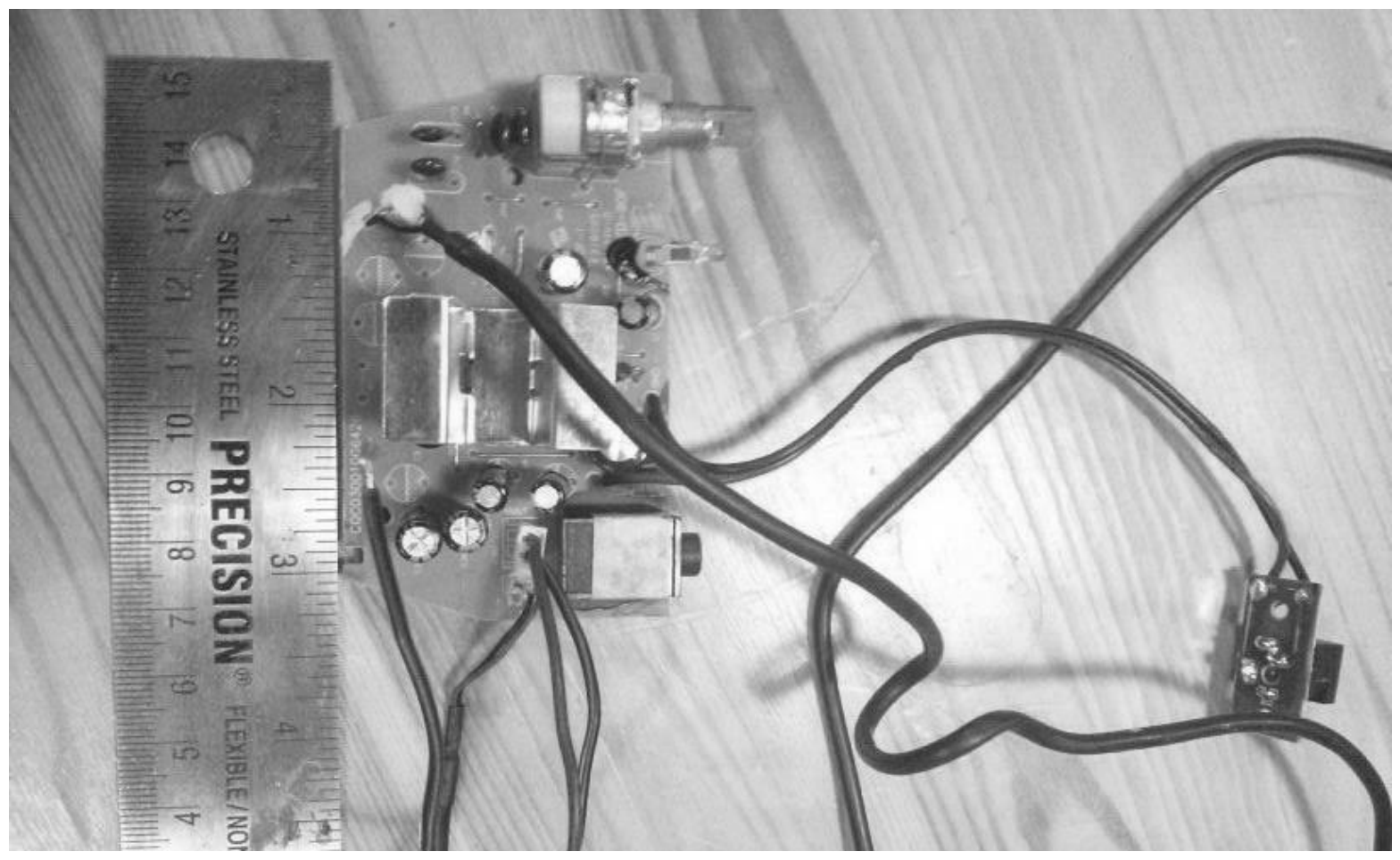

Figure 8. Customized PCB with amplifier.
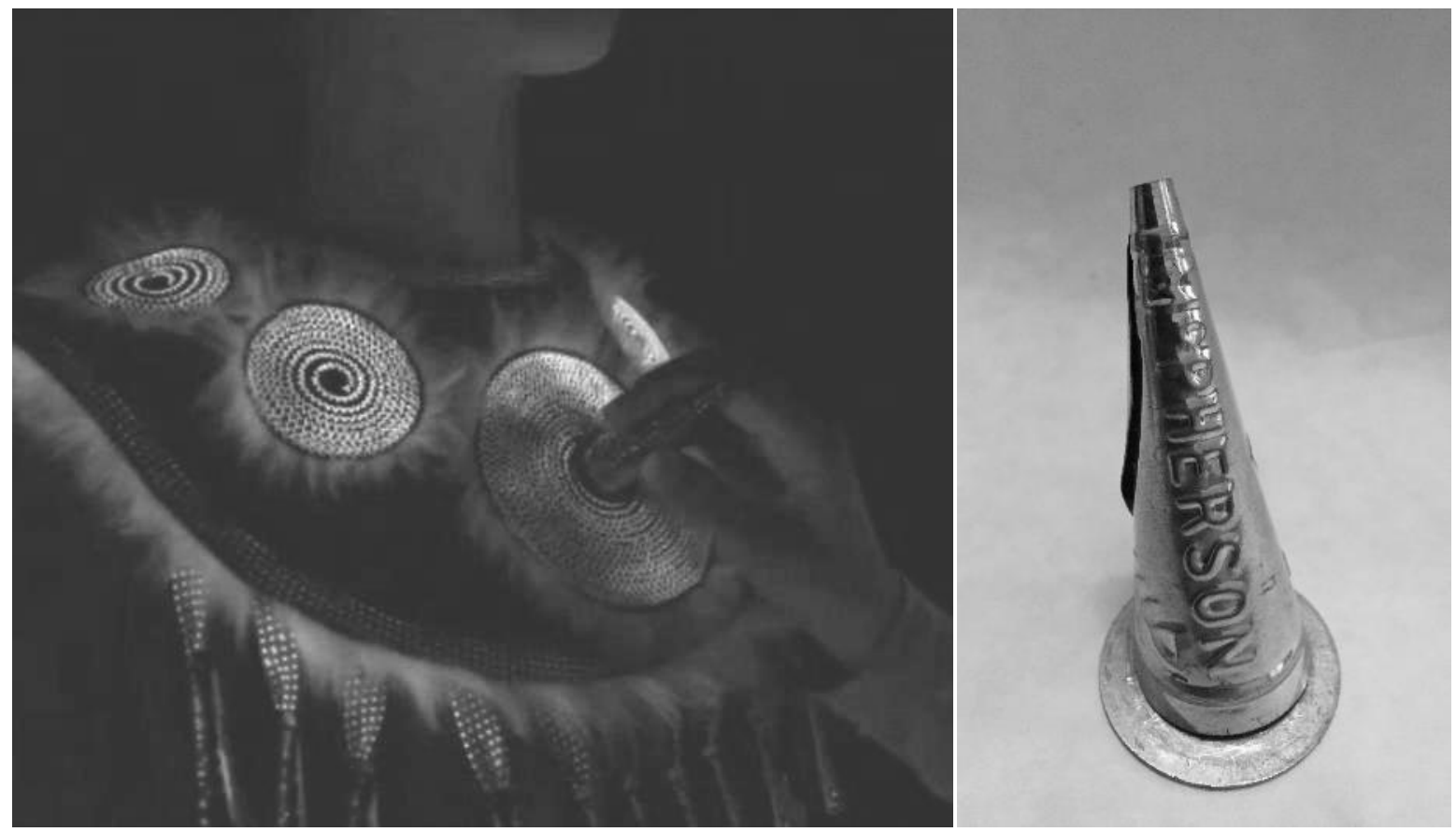

Figure 9. Handheld magnetic jingle cone. 


\section{References}

Antonelli, P. (2013). Why I brought Pac-Man to MoMA. TED Talk. Retrieved from https://www.ted.com/talks/paola_antonelli_why_i_brought_pacman_to_moma/transcript? language $=\mathrm{en}$

Armstrong, J. (2013). The hearing shirt—International Symposium on Wearable Computers 2013 application. Retrieved from http://jennilinarmstrong.com/blog/the-hearing-shirt

Arnold, J. (2012, June). Practice led research: Creative activity, academia debate, and intellectual rigour. Higher Education Studies, 2(2), 9-24. doi:10.5539/hes.v2n2p9

Bardeau, P. E. W. (2011). Definitive Seneca: It's in the word (J. Cardinal, Ed.). Salamanca, NY: Seneca-Iroquois Museum Publisher.

Belcher, M. (1991). Exhibitions in museums. Washington, DC: Smithsonian Institution Press.

Belcourt, C. (2010). Beadwork: First peoples' beading history and techniques. Owen Sound, ON: Ningwakwe Learning Press.

Benschop, R. (2009). All the names: Soundscapes, recording technology, and the historical sensation. In K. Bijsterveld \& J. van Dijck (Eds.), Sound souvenirs: Audio technologies, memory and cultural practices (pp. 182-198). Amsterdam, Netherlands: Amsterdam University Press.

Berzowska, J. (2010). XS Labs: Seven years of design research and experimentation in electronic textiles and reactive garments [Monograph]. Montréal, QC: XS Labs.

Borgdorff, H. (2011). The production of knowledge in artistic research. In M. Biggs \& H. Karlsson (Eds.), The Routledge companion to research in the arts (pp. 44-63). Oxon, UK: Routledge. 
Browner, T. (2002). Heartbeat of the people: Music and dance of the northern pow-wow. Chicago, IL: University of Illinois.

Buechley, L. (2007). LilyPad Arduino: E-textile construction kit version 2.0. Retrieved from http://web.media.mit.edu/ leah/grad_work/projects/e-textile_kit/e-textile_kit2.html

Canadian Reviewer. (2013, August 28). David Bowie retrospective show at AGO to offer immersive $3 D A V$ experience. Retrieved from http://www.canadianreviewer.com/cr/2013/8/28/david-bowie-retrospective-show-at-agoto-offer-immersive-3d.html

Cave, N. (2011, March). Meet me at the center of the Earth (Seattle Art Museum exhibition). Retrieved from http://soundsuitshop.com/scene/exhibitions/seattle-art-museum-march2011

Chatterjee, H. J. (Ed.). (2008). Touch in museums: Policy and practice in object handling. Oxford, UK: Berg.

Clavir, M. (2002). Preserving what is valued: Museums, conservation, and First Nations. Toronto, ON: UBC Press.

Collins, A. (2007). A tongue for an eye: Device challenges conventional vision (National Eye Institute news brief). Retrieved from http://www.nei.nih.gov/news/briefs/weihenmayer.asp Correll, N. (2012, June 24). "Flutter” wins first prizes at the ISWC design exhibition. Retrieved from http://correll.cs.colorado.edu/?p=2315

Court of Appeal Upholds Landmark Ruling on Rights of Métis. (2014, April 17). CBC News. Retrieved from http://www.cbc.ca/news/aboriginal/court-of-appeal-upholds-landmarkruling-on-rights-of-m\%C3\%A9tis-1.2613834 
Crazy Crow Trading Post. (1997). Jingle dress. Missouri River Brand Patterns. Pottsboro, TX: Crazy Crow Trading Post.

Denver Art Museum. (2013) Nick Cave—Sojourn. Jack Shainman Gallery. Retrieved from http://www.jackshainman.com/publications/cave/

Dewey, J. (1958). Art as experience. New York, NY: Capricorn Books.

Doward, J. (2014). vOICe: the soundscape headsets that allow blind people to 'see' the world. The Guardian News and Media Limited. Retrieved from http://www.theguardian.com/society/2014/dec/07/voice-soundscape-headsets-allow-blind-see

Entwistle, J., \& Wilson, E. (1998). The body clothed. In P. Wollen (Ed.), Addressing the century: 100 years of art and fashion (pp. 106-120). London, UK: Hayward Gallery.

Fitzhenry, M. (2013, January 29). CMHR to feature the most inclusive design in Canadian history. Council of Canadians with Disabilities. Retrieved from http://ccdonline.ca/en/humanrights/promoting/CMHR-press-release-29Jan2013

Geldard, F. (1972). The human senses. Toronto, ON: Wiley.

Grimmer, M. (2013, March 22). "David Bowie is" behind the scenes [Video file]. Retrieved from http://www.youtube.com/watch?v=ZKwfRsO7sV4\&list=PL3580D09534A34A24

Hill, R. (2014, February 8). Conversations about Indigenous visual culture wampum: Language and symbol (OCAD University lecture).

Hlady, M. (2008). Playing piano. Retrieved from http://www.marlahlady.com/sculpture/PlayingPiano.html

Huizinga, J. (1920). Het historisch museum (The historical museum). In Verzamelde Werken: vol. 2. Netherlands. 559-569. 
Hupfield, M. (2002). Jingle dress (Galerie Hugues Charbonneau installation). Retrieved from http://huguescharbonneau.com/en/maria-hupfield/

Interaccess. (n.d.). About. Retrieved from http://www.interaccess.org/about

Kim, P. (2014, November 29). Hearing impaired restaurant faces fines for adding wheelchair ramp. Global News. Retrieved from http://globalnews.ca/video/1699580/hearingimpaired-restaurant-faces-fines-for-adding-wheelchair-ramp

Klanten, R., Ehmann, S., \& Hanschke, V. (Eds.). (2011). A touch of code: Interactive installations and experiences. Berlin, Germany: Gestalten.

Klein, J. (2010). What is artistic research? Gegenworte, 23, 24-28. Retrieved from http://www.researchcatalogue.net/view/15292/15293

Lavallée, L. (2009). Practical application of an indigenous research framework and two qualitative indigenous research methods: Sharing circles and Anishnaabe symbol-based reflection. International Journal of Qualitative Methods, 8(1), 21-40. Retrieved from http://digitalcommons.ryerson.ca/islandora/object/RULA\%3A133

Leclerc, V. (2006). Accouphène tuxedo. Retrieved from http://www.xslabs.net/accouphene Mäkela, M. (2007). Knowing through making: The role of the artefact in practice-led research. Knowledge, Technology \& Policy, 20(3), 157-163. doi:10.1007/s12130-007-9028-2

McCarthy, J., \& Wright, P. (2004). Technology as experience. Cambridge, MA: MIT Press. McGinnis, R. (2012, May). Seeing through art: Blind visitors and the museum experience. Paper presented at the Symposium on Materiality \& Independence: Disability, Ability \& the Built Environment, Edmonton, AB. 
Millward, F. (2013). The practice-led fine art Ph.D.: At the frontier of what there is-An outlook on what there might be. Journal of Visual Art Practice, 12(2), 121-133. doi:10.1386/jvap.12.2.121_1

Milunovic, V. (2013a, September 18). Detail of the dress (Web log post). Retrieved from http://blog.europeanafashion.eu/2013/09/18/conservation-bridal-wedding-dress-museumof-applied-art-belgrad/

Milunovic, V. (2013, September 18). The wedding dress on display after conservation (Web log post). Retrieved from http://blog.europeanafashion.eu/2013/09/18/conservation-bridalwedding-dress-museum-of-applied-art-belgrad/

Ministry of Economic Development, Employment and Infrastructure. (2009). Accessibility for Ontarians with Disabilities Act, 2005. Retrieved from http://www.ontario.ca/laws/statute/05a11

Miralab. (2007). Haptex: Haptic sensing of virtual textiles. Retrieved from http://haptex.miralab.unige.ch/

Monsebraaten, L. (2015, February 24). Ontario to reduce enforcement of accessibility law. Toronto Star. Retrieved from http://www.thestar.com/news/canada/2015/02/24/ontarioto-reduce-enforcement-of-accessibility-law.html

Museo del Traje. (2013, September 18). Staff carefully handle its collection [Web log post]. Retrieved from http://blog.europeanafashion.eu/2013/07/

National Coalition for Vision Health. (2011). FAQs. Retrieved from visionhealth.ca

OCAD University. (2015). Accountability. Retrieved from http://www.ocadu.ca/about/accountability.htm 
Odegaard, N. (2004). The issue of pesticide contamination. In S. Odgen (Ed.), Caring for American Indian objects: A practical and cultural guide. Saint Paul, MN: Minnesota Historical Society Press.

O’Donnell, V., \& Wallace, S. (2014). First Nations, Métis and Inuit women (Statistics Canada publication no. 89-503-X). Retrieved from http://www.statcan.gc.ca/pub/89-503x/2010001/article/11442-eng.htm\#a2

Palmer, A. (2006). A bomb in the collection: Researching and exhibiting early twentieth century fashion. In C. Rogerson \& P. Garside (Eds.), The future of the twentieth century: Collecting, interpreting \& conserving modern materials (pp. 41-47). London, UK: Archetype.

Palmer, A. (2008). Untouchable: Creating desire and knowledge in museum costume and textile exhibition. Fashion Theory, 12(1), 31-64. doi:10.2752/175174108X268136

Perner-Wilson, H. (2011a). Actuators: Fabric speakers. In How to Get What You Want. Retrieved from http://www.kobakant.at/DIY/?p=2936

Perner-Wilson, H. (2011b). A kit of no parts: Recipes for materially diverse, functionally transparent and expressive electronics (Master's thesis). Massachusetts Institute of Technology, Cambridge, MA. Retrieved from http://web.media.mit.edu/ plusea/?p=265

Perner-Wilson, H. (2013, October 23). Example projects: Amplified pillow speaker. Retrieved from http://www.kobakant.at/DIY/?p=3857

Pheasant, K. (2006). The malady of the jingle dress (Aboriginal Curatorial Collective archives). Retrieved from http://www.aboriginalcuratorialcollective.org/research/jingle.html Proctor, N. (2009, April 25). Information architecture. In MuseumMobile Wiki. Retrieved from http://wiki.museummobile.info/museums-to-go/architecture 
Pyka, A. (1997). Informal networking. Technovation, 17(4), 207-220.

RNIB and Vocaleyes. (2003). Museums, galleries and heritage sites: Improving access for blind and partially sighted people. The Talking Images guide. Retrieved from Www.thetalkingwalls.co.uk/PDF/public_talkingimagesguide.pdf

Rabinovitch, S. (2011, May 24). The fashion world of Jean Paul Gaultier in Montreal [Web log post]. Retrieved from http://www.tourisme-montreal.org/blog/the-fashion-world-of-jeanpaul-gaultier-in-montreal/

Rowland, J. (2013). Multi-hex speaker array [Web log post]. Retrieved from http://www.jessrowland.com/art/electronicspaper-speakers/multihexspeakersmallpreview-jpg/

Ryerson University. (2015). Ryerson University at a glance. Retrieved from http://www.ryerson.ca/news/media/quickfacts/

Santoro, A. (2012, January 30). What is sonic fabric? Retrieved from http://sonicfabric.com/about.html

Schön, D. A. (1983). The reflective practitioner: How professionals think in action. New York, NY: Basic Books.

Scrivener, S. (2000). Reflections in and on action and practice in creative-production doctoral projects in art and design (Working Papers in Art and Design 1). Retrieved from http://tiny.cc/h44sxx

Shepherd, H. (2009). Inclusion and museum: Developing inclusive practice. British Journal of Special Education, 36(3), 140-145.

SHOWstudio. (2006a). The sound of clothes: Anechoic. Retrieved from http://showstudio.com/project/the_sound_of_clothes_anechoic 
SHOWstudio. (2006b). The sound of clothes: Synaesthesia. Retrieved from http://showstudio.com/project/the_sound_of_clothes_synaesthesia

Simms, S. J. (2005). A polluting concept of culture: Native artefacts contaminated with toxic preservatives. International Journal of Heritage Studies, 11(4), 327-339.

Simon, N. (2010). The participatory museum. Santa Cruz, CA: Museum 2.0.

Smith, H., \& Dean, R. T. (2009). Practice-led research, research-led practice in the creative arts. Edinburgh, Scotland: Edinburgh University Press.

Smith, L. T. (2012). Decolonizing methodologies: Research and indigenous peoples (2nd ed.). London, UK: Zed Books.

Sonicfabric.com. (2011, December 14). Sonic fabric: Galician-edition. Retrieved from http://www.sonicfabric.com/installation.html

Sonicfabric.com. (2011, December 14). Sonic fabric: Galician-edition. Retrieved from http://www.sonicfabric.com/installation.html

Steele, V. (2008). Museum quality: The rise of the fashion exhibition. Fashion Theory, 12(1), 730. doi: $10.2752 / 175174108 \times 268127$

Stoll, C. (1999). High-tech heretic. New York, NY: Doubleday.

Statistics Canada. (2009). Aboriginal Peoples in Canada in 2006: Inuit, Métis and First Nations, 2006 census. Retrieved from http://www12.statcan.ca/census-recensement/2006/as-sa/97558/index-eng.cfm

Statistics Canada. (2012). Population with a disability. Employment and social development in Canada. Retrieved from http://mieux-etre.edsc.gc.ca/mismeiowb/indicator.jsp?\&indicatorid $=40$ 
Strasdin, K. (2011, November 11). A royal wardrobe unlocked: Queen Alexandra 1863-1910. Paper presented at the 20th annual Veronika Gervers memorial lecture, Toronto, ON.

Sullivan, G. (2001). Artistic thinking as transcognative practice: A reconciliation of the processproduct dichotomy. Visual Arts Research, 27(1), 2-12.

Sullivan, G. (2006). Research acts in art practice. Studies in Art Education, 48(1), 19-35.

Textile Museum of Canada. (2010). Social fabric. Retrieved from http://www.textilemuseum.ca/socialfabric/Home.aspx

Thumping Threads Team Members. (2009, April 14). Feel the music: Thumping threads [Web log post]. Retrieved from http://thumpingthreads.blogspot.ca/

Vogel, P. (1999). Rhythmic sounds. Retrieved from http://www.bitforms.com/vogelschuelke/peter-vogel-rhythmic-sounds

Vogel, P. (2006). Interactive sonic \& light sculptures. Retrieved from http://www.bitforms.com/vogel-schuelke/peter-vogel-rhythmic-sounds

Vreeland, D. (1974). Romantic and glamorous Hollywood designs. New York, NY: The Costume Institute of the Metropolitan Museum of Art.

Wade, A. (2007). Balancing preservation and interaction in the museum setting. Greensboro, NC: University of North Carolina. 